ARTICLE

\title{
A severe leakage of intermediates to shunt products in acarbose biosynthesis
}

\author{
Qinqin Zhao (10) 1,2, Yuchang Luo ${ }^{1,2}$, Xin Zhang ${ }^{1,2}$, Qianjin Kang (iD) 1,2, Dan Zhang ${ }^{1,2}$, Lili Zhang ${ }^{3}$, \\ Linquan Bai (1) ${ }^{1,2,3 凶} \&$ Zixin Deng,
}

The $\alpha$-glucosidase inhibitor acarbose, produced by Actinoplanes sp. SE50/110, is a well-known drug for the treatment of type 2 diabetes mellitus. However, the largely unexplored biosynthetic mechanism of this compound has impeded further titer improvement. Herein, we uncover that 1-epi-valienol and valienol, accumulated in the fermentation broth at a strikingly high molar ratio to acarbose, are shunt products that are not directly involved in acarbose biosynthesis. Additionally, we find that inefficient biosynthesis of the amino-deoxyhexose moiety plays a role in the formation of these shunt products. Therefore, strategies to minimize the flux to the shunt products and to maximize the supply of the amino-deoxyhexose moiety are implemented, which increase the acarbose titer by 1.2 -fold to $7.4 \mathrm{~g} \mathrm{~L}^{-1}$. This work provides insights into the biosynthesis of the $\mathrm{C}_{7}$-cyclitol moiety and highlights the importance of assessing shunt product accumulation when seeking to improve the titer of microbial pharmaceutical products.

\footnotetext{
${ }^{1}$ State Key Laboratory of Microbial Metabolism, School of Life Sciences and Biotechnology, Shanghai Jiao Tong University, 200240 Shanghai, China. 2 Joint International Research Laboratory of Metabolic and Developmental Sciences, Shanghai Jiao Tong University, 200240 Shanghai, China. ${ }^{3}$ College of Life Science, Tarim University, Alar 843300 Xinjiang, China. ${ }^{凶}$ email: bailq@sjtu.edu.cn
} 
T he a-glucosidase inhibitor acarbose (1) is one of the prominent representatives of $\mathrm{C}_{7} \mathrm{~N}$-aminocyclitol-containing natural products and has been used for the clinical treatment of type 2 diabetes mellitus since it was introduced to the market in $1990^{1,2}$. This pseudosugar-containing oligosaccharide has competitive inhibitory activities toward intestinal maltase, sucrase, dextrinase, and glucoamylase, and thereby delays carbohydrate absorption and controls postprandial hyperglycemia ${ }^{1,3}$. It is produced in large-scale fermentation by Actinoplanes sp. obtained from rounds of mutagenesis and selection ${ }^{1,4,5}$. However, since type 2 diabetes mellitus becomes more prevalent worldwide, the market demand for $\mathbf{1}$ increases rapidly ${ }^{6}$, which encourages us to develop high-performance producers with improved productivity.

In recent years, comparative genome, transcriptome, and proteome analyses have provided systems-level understanding of Actinoplanes sp. SE50/110 and insights into the mechanisms of 1 overproduction $^{7-10}$. In addition, highly efficient genetic manipulation systems have been established for Actinoplanes sp. SE50/110 and are successfully used to delete melC2 (a tyrosinase gene) and $\operatorname{tre} Y$ (a maltooligosyltrehalose synthase gene), which eliminate the formation of eumelanin and the by-product component $\mathrm{C}$, respectively ${ }^{11-13}$. These advances in the omics analysis and genetic toolbox development have paved the way to genetically engineer Actinoplanes sp. SE50/110 to become a more efficient biofactory of $\mathbf{1}^{14-16}$.

However, attempts to substantially improve the titer of $\mathbf{1}$ also require a clear understanding of the biosynthetic pathway to 1 , as this information will enable identification of potential targets for gene manipulation and biosynthetic flux modulation. The biosynthetic gene cluster of $\mathbf{1}$ ( $a c b$ cluster) in Actinoplanes sp. comprises 22 open reading frames responsible for the biosynthesis and export of 1 and sugar metabolism ${ }^{1,17,18}$ (Fig. 1a; Supplementary Table 1). The structure of $\mathbf{1}$ consists of three moieties: an unsaturated $\mathrm{C}_{7}$-cyclitol, an amino-deoxyhexose, and a maltose. The biosynthesis of the $\mathrm{C}_{7}$-cyclitol moiety has been demonstrated to be initiated by the cyclization of sedo-heptulose-7-phosphate (sedo-heptulose-7-P, 2) by AcbC to give 2-epi-5-epi-valiolone (3), which is subsequently phosphorylated by AcbM and isomerized by AcbO to give intermediate 5-epi-valiolone-7-P (4) ${ }^{19-21}$. On the other hand, the amino-deoxyhexose moiety has been shown to derive from glucose-1-P (5) catalyzed by D-glucose-1-P thymidylyltransferase (AcbA, abbreviated as G-1-PT) and dTDP-Dglucose 4,6-dehydratase (AcbB, 4,6-DH) to give dTDP-4-keto-6deoxy-D-glucose (6), followed by transamination catalyzed by AcbV to give dTDP-4-amino-4,6-dideoxy-D-glucose $(7)^{1,22}$. Further downstream, the pathway to $\mathbf{1}$ has been proposed to involve modifications of $\mathbf{4}$, condensation of the $\mathrm{C}_{7}$-cyclitol moiety with 7 , and the attachment of maltose to form 1 , without experimental evidence (Fig. 1b) $)^{1,21,23,24}$. The limited knowledge of the biosynthetic pathway to $\mathbf{1}$ has made it difficult to identify critical biosynthetic bottlenecks and to prioritize engineering strategies for the overproduction of $\mathbf{1}$.

In the present study, we identified two shunt products that are derived from the biosynthetic pathway of $\mathbf{1}$ in the fermentation broth of Actinoplanes sp. SE50/110. Upon systematic investigation of these products and their modes of formation, we further clarify the biosynthetic pathway to the $\mathrm{C}_{7}$-cyclitol moiety in $\mathbf{1}$. Subsequently, we employ multiple metabolic engineering strategies to modulate the flux between the $\mathrm{C}_{7}$-cyclitol and the aminodeoxyhexose moieties and are able to substantially increase the titer of $\mathbf{1}$ and decrease the accumulations of the shunt products.

\section{Results}

Discovery of two main shunt products of acarbose. During the high-performance liquid chromatography (HPLC) analysis of the fermentation broth of Actinoplanes sp. SE50/110, we observed a predominant peak (P-1) at a retention time of $7.5 \mathrm{~min}$, while acarbose (1) appeared at $20.8 \mathrm{~min}$ (Fig. 2a). To examine whether peak P-1 was related to 1 , the whole $a c b$ cluster $(32.2 \mathrm{~kb})$ was deleted in Actinoplanes sp. SE50/110, and the mutant was named QQ-3. Both P-1 and 1 disappeared in the fermentation broth of mutant QQ-3, and were restored by trans-complementation of the $a c b$ cluster cloned on fosmid pLQ666 ${ }^{13}$ (Fig. 2a). The simultaneous disappearance and restoration of P-1 and 1 established a correlation between these two peaks.

Through purification and structural elucidation by nuclear magnetic resonance (NMR) spectroscopy, 1-epi-valienol (8) and valienol (9) were confirmed to be present in P-1 (Supplementary Figs. 1-10; Supplementary Table 2; Fig. 2b). To quantify the amounts in fermentation broth, both compounds were derivatized by $\mathrm{N}, \mathrm{O}$-bis(trimethylsilyl)trifluoroacetamide (BSTFA) and analyzed by gas chromatography-quadrupole mass spectrometry (GC-QMS) (Supplementary Fig. 11). Strikingly, 8 and 9 were found to be accumulated at $2.5 \mathrm{~g} \mathrm{~L}^{-1}(14.2 \mathrm{mM})$ and $1.6 \mathrm{~g} \mathrm{~L}^{-1}(9.1 \mathrm{mM})$, respectively, in a 4-day fermentation broth of Actinoplanes sp. SE50/110, whereas the titer of $\mathbf{1}$ was only $3.1 \mathrm{~g} \mathrm{~L}^{-1}(4.8 \mathrm{mM})$. To investigate whether 8 and 9 were hydrolytic products of $\mathbf{1}, \mathbf{1}$ was fed to the $a c b C$-deleted mutant QQ-4 (Supplementary Fig. 12). However, neither 8 nor 9 were detected, suggesting that they are not derived from $\mathbf{1}$ (Supplementary Fig. 13).

To examine whether 8 and 9 could be incorporated into 1, both compounds and 2-epi-5-epi-valiolone (3), the precursor of the $\mathrm{C}_{7}$-cyclitol moiety in $\mathbf{1}^{19,20}$, were fed to the mutant QQ-4. While feeding with 3 resulted in substantial accumulation of $\mathbf{1}$, no production of 1 was detected after feeding with 8 or 9 (Fig. 2c), suggesting that $\mathbf{8}$ and $\mathbf{9}$ are shunt products, not the precursors of or intermediates in $\mathbf{1}$ biosynthesis.

Dephosphorylation of the proposed $\mathrm{C}_{7}$-cyclitol intermediates. On the basis of the proposed biosynthetic pathway to $\mathbf{1}^{1}$ (Fig. 1b), it may be postulated that $\mathbf{8}$ is derived from 1-epi-valienol-7-P (10) by a phosphatase. In order to biochemically validate this hypothesis, we prepared compound $\mathbf{1 0}$ by incubating $\mathbf{8}$ with ValC, a known $\mathrm{C}_{7}$-cyclitol kinase involved in the biosynthesis of validamycin in Streptomyces hygroscopicus var. jinggangensis $5008^{25}$. ValC was able to phosphorylate $\mathbf{8}$, and the product $\mathbf{1 0}$ $\left(\mathrm{m} / z=255.0308[\mathrm{M}-\mathrm{H}]^{-}\right)$was confirmed. Moreover, ValC was able to catalyze the phosphorylation of 9 to give valienol-7-P (11) (Supplementary Figs. 14-16; Supplementary Table 3).

With both compounds $\mathbf{1 0}$ and $\mathbf{1 1}$ in hand, we decided to identify the enzymes that are responsible for the dephosphorylation. The AcbJ protein showed high sequence similarity to hydrolases of the haloacid dehalogenase-like (HAD) superfamily and was initially hypothesized to be responsible for the dephosphorylation of 10 (Supplementary Table 1). The gene was expressed in Escherichia coli, and the product was purified. AcbJ was able to efficiently dephosphorylate 10 to $\mathbf{8}$ (Fig. 3a). To further examine the function of AcbJ in vivo, the $a c b J$ gene was inactivated in Actinoplanes sp. SE50/110 to give a mutant QQ-5. Compared with Actinoplanes sp. SE50/110, QQ-5 showed a 76.4\% decrease of phosphatase activity, implying that AcbJ plays a primary role in the dephosphorylation of 10. In addition, inactivation of $a c b J$ abolished the production of $\mathbf{1}$, which was partially restored by the trans-complementation of a cloned $a c b J$ (Supplementary Fig. 17), indicating that AcbJ is essential for the biosynthesis of 1 . Moreover, the slight decrease in the accumulation of $\mathbf{8}$ and the residual phosphatase activity suggested that other enzymes, possibly encoded by genes located outside the $a c b$ cluster, may participate in the dephosphorylation of $\mathbf{1 0}$. 
a

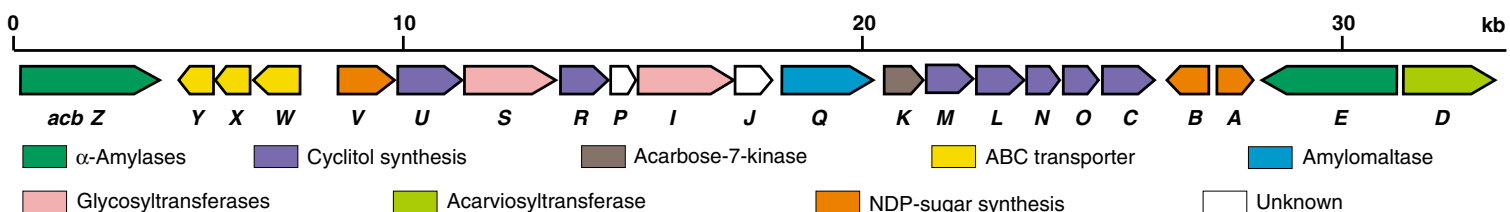

b
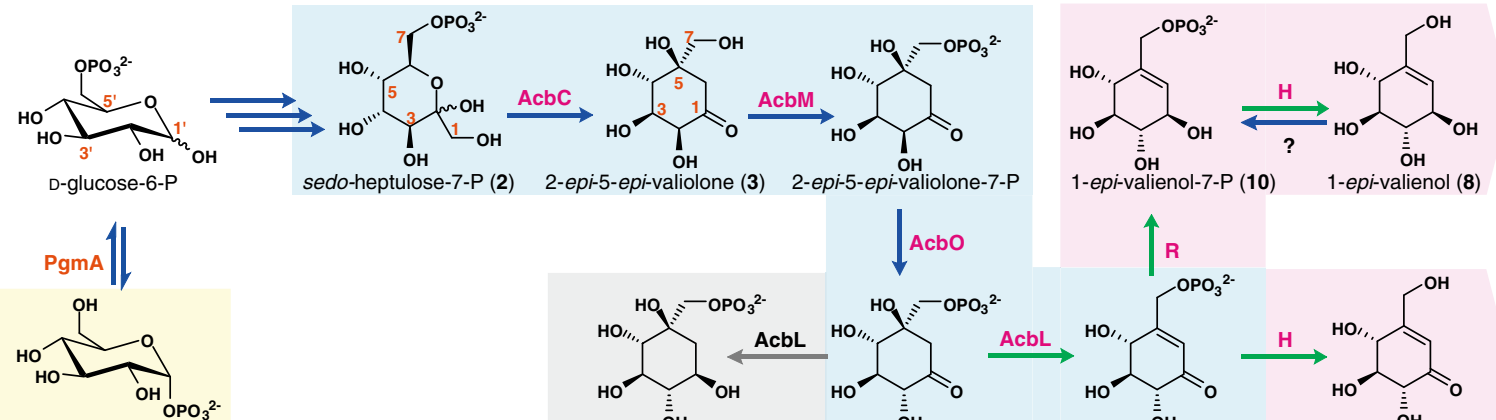

D-glucose-1-P (5)

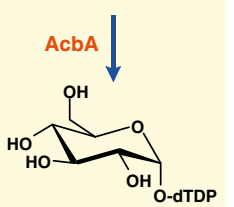

dTDP-D-glucose

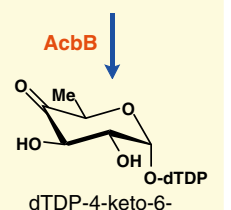

deoxy-D-glucose (6)

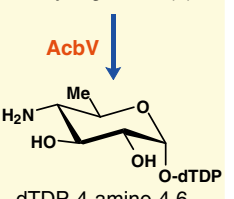

dTDP-4-amino-4,6-

dideoxy-D-glucose (7)

1-epi-valienol-7-P (10)

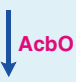

(-)

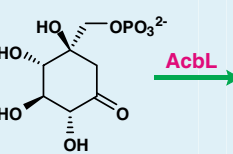

5-epi-valiolol-7-P

5-epi-valiolone-7-P (4)<smiles>C1CCCC1</smiles>

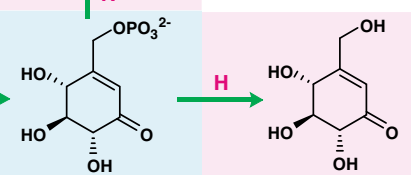

valienone-7-P (12)

valienone (13)

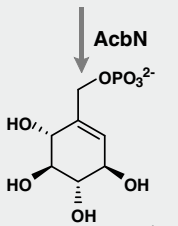

1-epi-valienol-7-P (10)

AcbUJ?

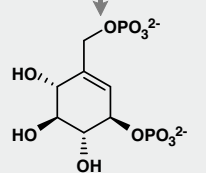

-epi-valienol-1,7-diP
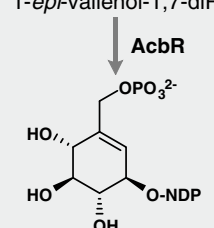

NDP-1-epi-valienol-7-P
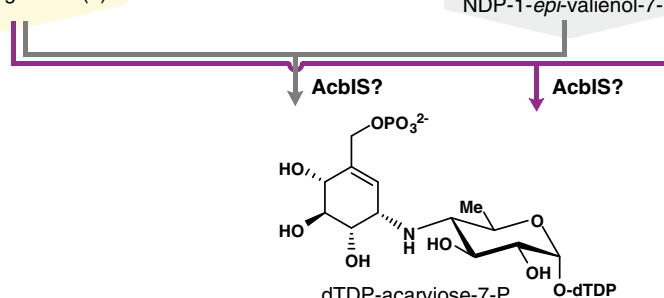

$\longrightarrow$ Previously proved biosynthetic steps

Previously proposed biosynthetic steps

..... Unconfirmed biosynthetic step

$\longrightarrow$ Confirmed biosynthetic steps in this work

dTDP-acarviose-7-P O-dTDP

$\longrightarrow$ Update of the previously proposed biosynthetic steps according to this work

$\mathrm{C}_{7}$-cyclitol moiety biosynthetic pathway

Amino-deoxyhexose moiety biosynthetic pathway

Previously proposed biosynthetic pathway

Shunt pathways

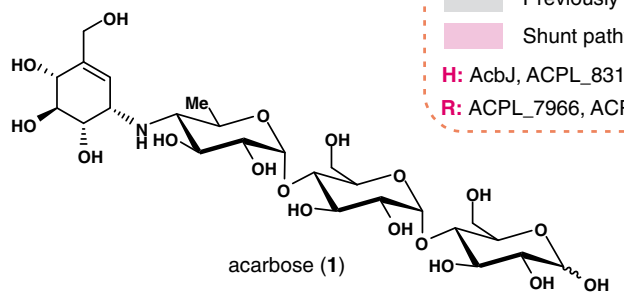

Fig. 1 The biosynthesis of acarbose in Actinoplanes species. a The biosynthetic gene cluster of acarbose (1) (acb cluster, GenBank accession no. Y18523.4). b The biosynthetic pathway to 1, including previously determined biosynthetic steps (blue arrow), possible conversion without experimental confirmation (gray dashed arrow), previously proposed biosynthetic steps (gray arrow), confirmed biosynthetic steps in this work (green arrow), and update of the previously proposed biosynthetic steps according to this work (purple arrow). The biosynthetic pathways to the $\mathrm{C}_{7}$-cyclitol moiety, the amino-deoxyhexose moiety, and the shunt products are highlighted in blue, yellow, and plum, respectively, and the previously proposed biosynthetic pathway to the $\mathrm{C}_{7}$-cyclitol moiety is highlighted in gray. 

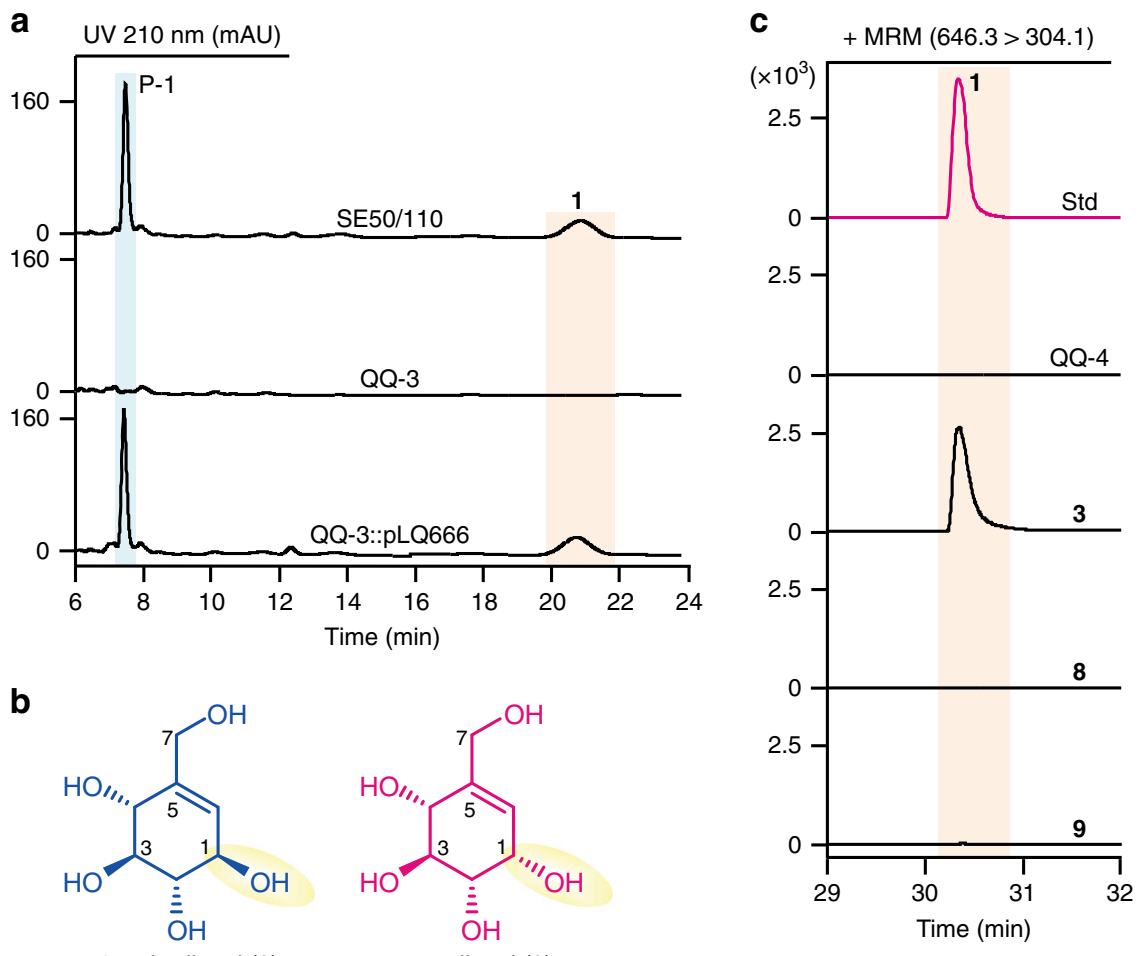

b
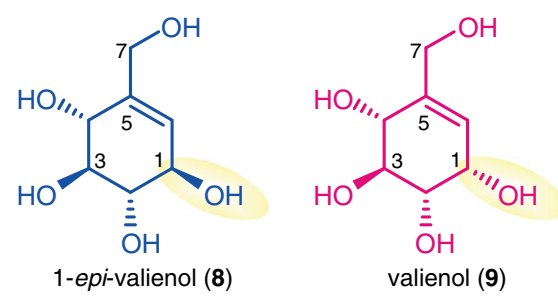

Fig. 2 Discovery and identification of shunt products accumulated in the fermentation broth of Actinoplanes sp. SE50/110. a HPLC profiles of the parent strain Actinoplanes sp. SE50/110 (abbreviated as SE50/110), the $\triangle a c b$ mutant QQ-3 and the complemented mutant QQ-3::pLQ666. b Structures of 1-epivalienol (8) and valienol (9). c HPLC-QQQ/MS analysis of $\mathbf{1}$ after feeding with $\mathbf{3}$ (as positive control), 8 or $\mathbf{9}$ to the $\triangle a c b C$ mutant QQ-4, and fermentation of QQ-4 without feeding was set as negative control. The standard (abbreviated as std) of $\mathbf{1}$ was also analyzed. Source data underlying Fig. 2c are provided as a Source Data file.

To further identify other potential hydrolases, four candidate proteins (ACPL_8310, ACPL_8309, ACPL_6858, and ACPL_7709) with sequence homology to AcbJ were identified using the BLASTP program $^{26}$, and four HAD superfamily hydrolases (ACPL_2834, ACPL_1897, ACPL_560, and ACPL_7310) with high expression levels were selected according to the transcriptomic analysis of Actinoplanes sp. SE50/1108 (Supplementary Table 4). Upon incubation of each purified enzyme with 10, ACPL_8310, ACPL_2834, and ACPL_7709 were found to catalyze the dephosphorylation of 10 (Fig. 3a; Supplementary Fig. 18a), whereas ACPL_8309 showed weak catalytic activity (Supplementary Fig. 19a). Interestingly, 11 was also dephosphorylated to 9 by these five enzymes having activities toward 10 (Fig. 3b; Supplementary Figs. $18 \mathrm{~b}$ and $19 \mathrm{~b}$ ). Moreover, the deletion of $a c b J$ resulted in a $66.9 \%$ decrease of the accumulation of 9 (Supplementary Fig. 17c).

Further clarification of $\mathrm{C}_{7}$-cyclitol moiety biosynthesis. Since $\mathrm{ValC}$ is capable of phosphorylating $\mathbf{8}$ and $\mathbf{9}$ to $\mathbf{1 0}$ and $\mathbf{1 1}$ in vitro (Supplementary Fig. 14), respectively, an attempt to recycle $\mathbf{8}$ and 9 in vivo was performed in mutant QQ-4:: valC with the gene valC integrated in the chromosome. Based on the previously proposed biosynthetic pathway ${ }^{1}$ (Fig. 1b), the recycling of $\mathbf{8}$ was expected to lead to the production of $\mathbf{1}$ via the formation of the putative intermediate 10. However, our feeding experiments with $\mathbf{8}$ and 9 revealed that only $\mathbf{9}$ can rescue the production of $\mathbf{1}$ in the QQ-4:: valC mutant, suggesting that $\mathbf{1 1}$ is a true intermediate for the biosynthesis of 1 (Supplementary Fig. 20). To further clarify the biosynthetic pathway of $\mathbf{1}$, we decided to revisit the proposed biochemical function of AcbL, which was annotated as a cyclitol dehydrogenase and proposed to catalyze the reduction of the C-1 carbonyl group of $\mathbf{4}$ to generate 5-epi-valiolol-7- $\mathrm{P}^{1}$ (Supplementary Table 1; Fig. 1b). Using sequential catalysis of AcbM and
AcbO, compound 4 was prepared from $3^{21}$, which was isolated from the fermentation broth of $S$. hygroscopicus var. jinggangensis TL01 $\Delta$ valD $^{27}$ (Supplementary Fig. 21). Interestingly, when the purified AcbL was incubated with $\mathbf{4}$ and NADPH (nicotinamide adenine dinucleotide phosphate), a product with a deprotonated molecular ion of $m / z=253.0121$ was detected (Fig. $4 a$ ), which was consistent with the calculated $\mathrm{m} / z$ of valienone-7-P $(\mathbf{1 2}$, calc. $\left.253.0119[\mathrm{M}-\mathrm{H}]^{-}\right)^{25}$ (Supplementary Fig. 22a). Furthermore, the reaction mixture was incubated with the phosphatase AcbJ, and the dephosphorylated product was identified as valienone (13) (Fig. 4b; Supplementary Figs. 22, 23). AcbL preferred to use NADPH as a cofactor in this reaction (Supplementary Fig. 24). These results indicated that AcbL catalyzes the dehydration of 4 to 12 (Fig. 1b). In addition, a trace amount of $13\left(\sim 20 \mathrm{mg} \mathrm{L}^{-1}\right)$, likely derived from the intermediate $\mathbf{1 2}$ by dephosphorylation, was detected in the fermentation broth of Actinoplanes sp. SE50/ 110. Compound $\mathbf{1 3}$ is proposed to be another shunt product in acarbose biosynthesis (Fig. 1b; Supplementary Fig. 25).

AcbN, annotated as a NADPH-dependent oxidoreductase of the short-chain dehydrogenase family (Supplementary Table 1), was proposed to catalyze the reduction of $\mathbf{1 2}$. To test this hypothesis, $\mathbf{1 2}$ was prepared from the phosphorylation of $\mathbf{1 3}$ by ValC (Supplementary Fig. 26; Supplementary Table 5). When purified AcbN was incubated with 12 and NADPH, a product with a deprotonated molecular ion at $m / z=255.0319$ was detected, which is the same as

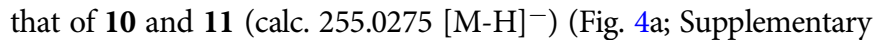
Fig. 14c). To determine the configuration of AcbN-catalyzed product, AcbJ was subsequently added, and the dephosphorylated product was confirmed to be 9 by GC-QMS (Fig. 4b), suggesting that AcbN catalyzes the conversion of 12 to 11 (Fig. 1b). Furthermore, the product was purified and confirmed as $\mathbf{1 1}$ by NMR spectroscopy (Supplementary Figs. 27-30; Supplementary 

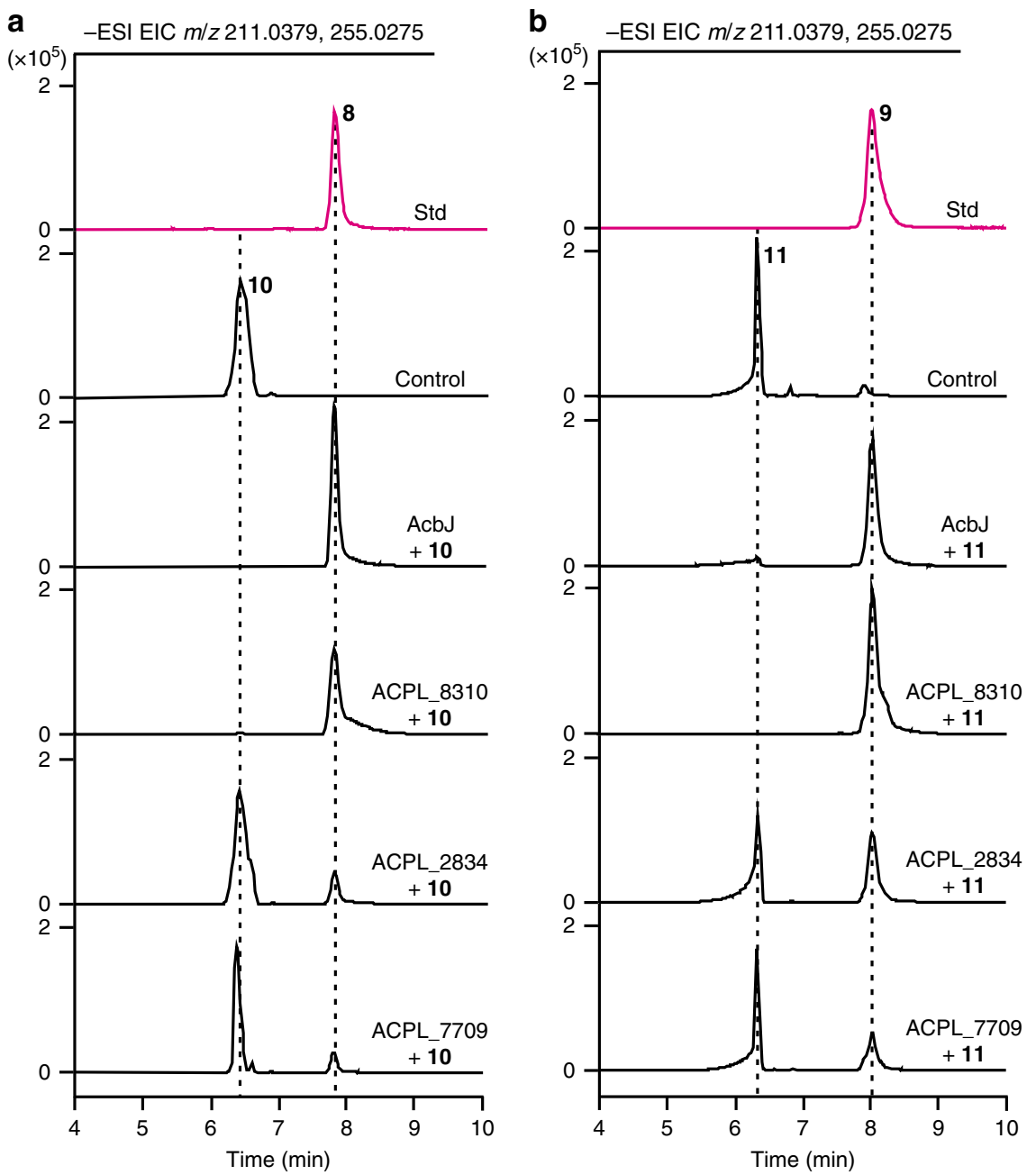

Fig. 3 Identification of hydrolases involved in the dephosphorylation of $\mathbf{1 0}$ and $\mathbf{1 1}$. $\mathbf{a}, \mathbf{b}$ HPLC-TOF/MS analysis of the dephosphorylated products of 10 and $\mathbf{1 1}$ catalyzed by AcbJ, ACPL_8310, ACPL_2834, or ACPL_7709 in vitro, and the reaction without enzyme was set as a negative control. The standards (std) of 8 and $\mathbf{9}$ were also analyzed. All of the chromatograms show the simultaneous extraction of calculated ions $\mathrm{m} / z=211.0379[\mathrm{M}+\mathrm{Cl}]-$ for $\mathbf{8}$ and $\mathbf{9}$ and $m / z=255.0275[\mathrm{M}-\mathrm{H}]^{-}$for $\mathbf{1 0}$ and $\mathbf{1 1}$. Source data are provided as a Source Data file.

Table 5). NADPH is also the preferential cofactor in this reaction (Supplementary Fig. 31). To test whether AcbN catalyzes the reversible reaction, the purified $A c b N$ was incubated with $\mathbf{1 1}$ and $\mathrm{NADP}^{+}$. The reaction gave a large amount of $\mathbf{1 2}$ (Fig. $4 \mathrm{a}, \mathrm{b}$ ). However, no product was detected in the incubation of AcbN with 10 (Supplementary Fig. 32), suggesting that AcbN specifically recognizes 11 as a substrate. These results indicated that $A c b N$ is responsible for the interconversion between $\mathbf{1 2}$ and 11, and the shunt product $\mathbf{9}$ is branched from $\mathbf{1 1}$ through dephosphorylation. Overall, the five biosynthetic steps from 2 to 11 , leading to the formation of the $\mathrm{C}_{7}$-cyclitol moiety of $\mathbf{1}$, have been completely illustrated (Fig. 1b; Supplementary Fig. 33).

Elucidation of the biosynthesis of 1-epi-valienol. Since AcbN has been shown to specifically catalyze the reduction of $\mathbf{1 2}$ to $\mathbf{1 1}$, the shunt product $\mathbf{1 0}$ is likely to be derived from $\mathbf{1 2}$ by other oxidoreductases. To prove this hypothesis, the gene $a c b N$ was inactivated in Actinoplanes sp. SE50/110. The mutant, SE50/ $110 \triangle a c b N$, was not able to produce 9 and $\mathbf{1}$, but still produced a large amount of $\mathbf{8}$, indicating that $\mathbf{8}$ and $\mathbf{9}$ are synthesized via different routes (Supplementary Fig. 34). Subsequently, the crude cell-free extracts (CFEs) of mutant SE50/110 $\triangle a c b N$, QQ-3 (with $a c b$ cluster deleted), and Actinoplanes sp. SE50/110 were individually incubated with 12, and large amounts of 10 and/or 11 $\left(\mathrm{m} / z=255.028\right.$, calc. 255.0275 [M-H $\left.^{-}\right)$were detected (Fig. 5a; Supplementary Fig. 14c). The products were then dephosphorylated with AcbJ and analyzed by GC-QMS, and those from the SE50/110 $\triangle a c b N$ and QQ-3 CFE reaction mixtures gave 8, while that from the Actinoplanes sp. SE50/110 CFE reaction mixture gave 8 and 9 (Fig. 5b). Furthermore, the product of the QQ-3 CFE reaction was purified and confirmed as $\mathbf{1 0}$ by NMR spectroscopy (Supplementary Figs. 35-39; Supplementary Table 6). Since there was no ORF in the $a c b$ cluster annotated as an oxidoreductase except for $a c b N$ (Supplementary Table 1), 12 is deduced to be reduced to $\mathbf{1 0}$ by enzymes encoded by genes located outside the $a c b$ cluster.

To identify the enzymes that are involved in this reaction, total proteins from the cell lysate of QQ-3 were fractionated into 34 fractions by anion exchange chromatography (Supplementary Fig. 40a), and each fraction was assayed for the reductase activity using 12 as substrate. Fractions 15 (F15) and 24 (F24) with highest reductase activity in the presence of NADPH and boiled cell-free extract (BCF), respectively, and F10 with undetectable activity were subjected to proteomic analysis (Supplementary Fig. 40b). The unique putative oxidoreductases with high reliability and abundance (Unique PepCount $\geq 4$ ) in F15 and F24 were biochemically characterized (Supplementary Fig. 40c; Supplementary Table 7). We discovered that the ACPL_7966, 

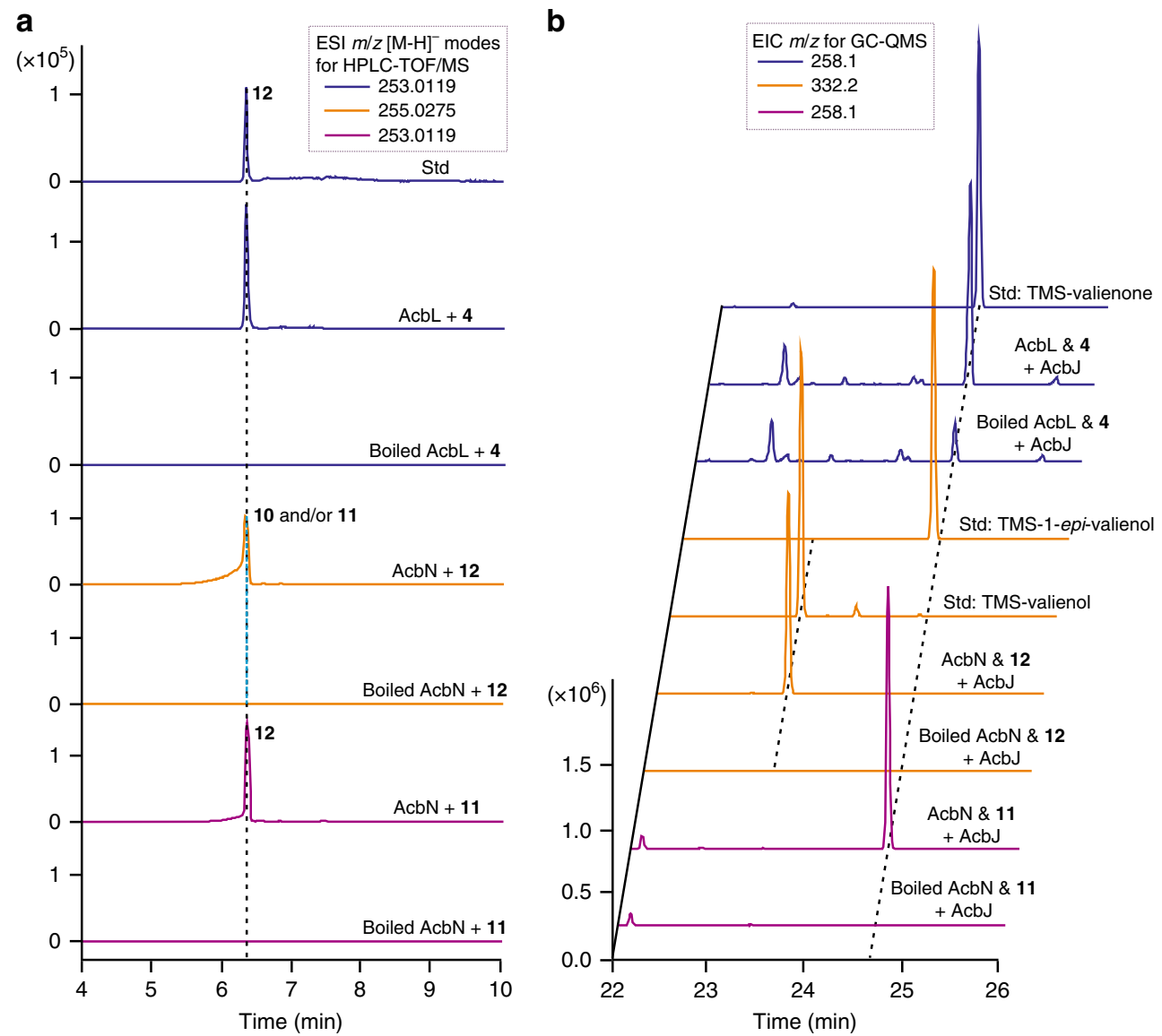

Fig. 4 Further clarification of the $\mathbf{C}_{\mathbf{7}}$-cyclitol moiety biosynthetic pathway by characterizing AcbL- and AcbN-catalyzed conversions. a HPLC-TOF/MS analysis of the standard (std) of $\mathbf{1 2}$ and the reaction products of AcbL with $\mathbf{4}$, boiled AcbL with $\mathbf{4}$, AcbN with 12, boiled AcbN with 12, AcbN with $\mathbf{1 1}$, and boiled AcbN with 11. All the chromatograms show the extraction of corresponding calculated ions. $\mathbf{b}$ GC-QMS confirmation of the reaction products after dephosphorylation by AcbJ. AcbJ was added to the reaction products of $A c b L \& \mathbf{4}$, boiled AcbL \& 4, AcbN \& 12, boiled AcbN \& 12, AcbN \& 11, and boiled $A c b N \&$ 11. The standards (std) $\mathbf{1 3}, \mathbf{8}$, and $\mathbf{9}$ were also analyzed by GC-QMS after derivatization by BSTFA. All the chromatograms show the extraction of corresponding unique product ions. TMS is the abbreviation of trimethyl silicyl. For more details, see also Supplementary Figs. 11, 23. Source data are provided as a Source Data file.

ACPL_7511, and ACPL_5736 proteins in F15 and the ACPL_7667, ACPL_5740, and ACPL_4412 proteins in F24 showed reductase activity (Fig. 5a, b; Supplementary Table 7). These results confirmed that the shunt product $\mathbf{8}$ is branched from the biosynthetic intermediate $\mathbf{1 2}$ by sequential reduction and dephosphorylation (Fig. 1b; Supplementary Fig. 41).

Minimization of the metabolic flux toward the shunt products. The parent strain Actinoplanes sp. SE50/110 produces $4.1 \mathrm{~g} \mathrm{~L}^{-1}$ $(23.3 \mathrm{mM})$ of 8 and 9 and only $3.1 \mathrm{~g} \mathrm{~L}^{-1}(4.8 \mathrm{mM})$ of 1 after 4day fermentation (Supplementary Fig. 11), suggesting that the shunt pathways lead to a severe leakage of $\mathrm{C}_{7}$-cyclitol intermediates 11 and 12, which were considered as the key metabolic branch points in the biosynthesis of $\mathbf{1}$. Therefore, we proposed that the titer of 1 might be improved by diverting the metabolic flux of the shunt products toward phosphorylated $\mathrm{C}_{7}$-cyclitols (11 and 12) using metabolic engineering strategies, such as reducing the phosphatase activity, decreasing the conversion of $\mathbf{1 2}$ to the shunt product 10, and enhancing the conversion of $\mathbf{1 2}$ to intermediate 11 (Fig. 6a).

Even though AcbJ is the primary phosphatase involved in the dephosphorylation of $\mathbf{1 0}$ and $\mathbf{1 1}$, it is essential for the biosynthesis of 1 and therefore cannot be deleted (Supplementary Fig. 17). Alternatively, the expression level of $a c b J$ can be tuned down using a weaker promoter. Cassettes of $a c b J$ under the control of promoters with different strengths were individually introduced into the $a c b J$-deleted mutant QQ-5. Using the $k a s O p^{*}$ promoter, the phosphatase activity was reduced to $54.2 \%$ of that of the control strain SE50/110::pSET152, which resulted in increased titer and productivity (ratio of titer of $\mathbf{1}$ and dry cell weight) of $\mathbf{1}$ (Supplementary Fig. 42). The cassette $k a s O p^{*}-a c b J$ was then introduced into the chromosome of QQ-7 (a QQ-5 derivative with the hydrolase gene $A C P L \_8310$ deleted and the expression of the hydrolase gene $A C P L \_2834$ down-regulated by replacing its native promoter with a weaker promoter $W V p$ ) (Supplementary Fig. 43) by homologous recombination. The resulting strain QQ-8 showed an $18.8 \%$ and a $30.3 \%$ increase in titer and productivity of 1, respectively (Fig. 6b; Supplementary Fig. 44a). The strain also showed a $7.1 \%$ and a $58.3 \%$ decrease in the accumulations of $\mathbf{8}$ and 9, respectively (Fig. 6c), a 59.8\% decrease in phosphatase activity (Supplementary Fig. 44b), a 1.2-fold increase in the total amounts of endogenous 10 and 11 (Supplementary Fig. 44c), and an $8.8 \%$ decrease in biomass (Supplementary Fig. $44 \mathrm{~d}$ ).

In order to decrease the conversion of $\mathbf{1 2}$ to $\mathbf{1 0}$ and promote the conversion of $\mathbf{1 2}$ to $\mathbf{1 1}, a c b N(A N)$ was overexpressed in QQ12 (a QQ-8 derivative with successive deletion of the oxidoreductase genes $A C P L \_7966, A C P L \_7511, A C P L \_5736$, and $\left.A C P L \_7667\right)$ (Fig. 6a). Although no obvious titer improvement of $\mathbf{1}$ and change in biomass were observed, the accumulation of 9 was increased by $30.0 \%$, whereas the accumulation of 8 was 

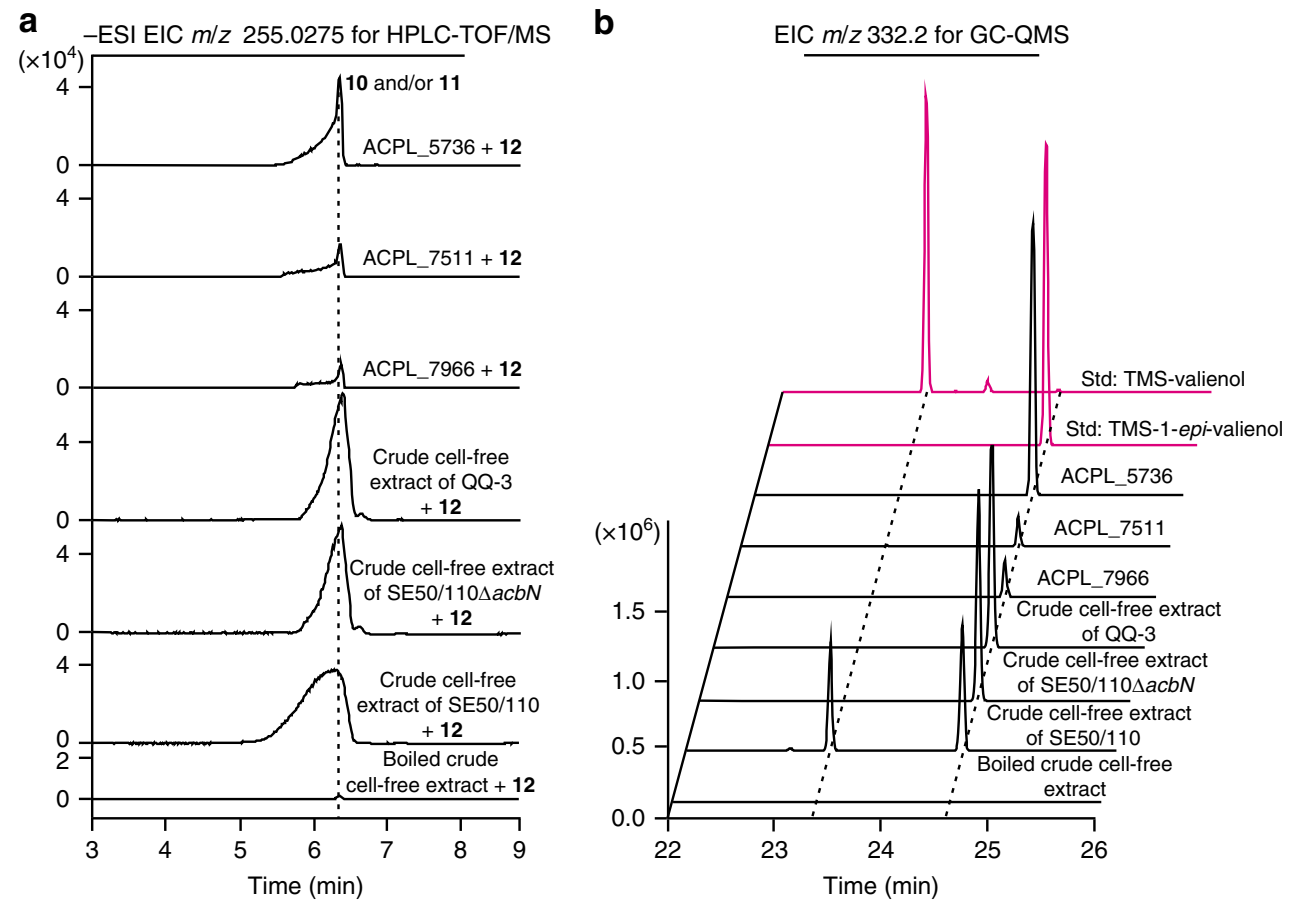

Fig. 5 Elucidation of the biosynthetic pathway to the shunt product 8. a HPLC-TOF/MS analysis of the reduction products of $\mathbf{1 2}$ by the crude cell-free extract of Actinoplanes sp. SE50/110 (abbreviated as SE50/110), SE50/110 $\Delta a c b N$ and QQ-3, purified ACPL_7966, ACPL_7511 and ACPL_5736 proteins, and the reaction with boiled crude cell-free extract (BCF) was set as a negative control. All the chromatograms show the extraction of calculated ion $m / z=$ $255.0275[\mathrm{M}-\mathrm{H}]^{-}$. $\mathbf{b}$ GC-QMS confirmation of the configuration of the reaction products after dephosphorylation by AcbJ. AcbJ was added to the reaction products of crude cell-free extract of Actinoplanes sp. SE50/110, SE50/110 $\Delta a c b N$, and QQ-3, purified ACPL_7966, ACPL_7511, ACPL_5736 proteins, and control reaction. The standards (std) $\mathbf{8}$ and $\mathbf{9}$ were also analyzed by GC-QMS after derivatization by BSTFA. For more details, see also Supplementary Fig. 11. All the chromatograms show the extraction of unique product ion $m / z=332$.2. TMS is the abbreviation of trimethyl silicyl. Source data are provided as a Source Data file.

decreased by $5.8 \%$ (Fig. 6b, c; Supplementary Fig. 44e), suggesting that the overexpression of $a c b N$ could improve the competing utilization of $\mathbf{1 2}$ for the biosynthesis of $\mathbf{1 1}$.

Maximizing the biosynthesis of amino-deoxyhexose moiety. Even though the abovementioned engineering strategies led to a moderate titer increase of $\mathbf{1}$, intracellular phosphorylated $\mathrm{C}_{7}$ cyclitols, including the intermediate 11, were substantially accumulated in the mutant QQ-12 (Fig. 6a-c; Supplementary Fig. 44), implying that an inefficient utilization of these $\mathrm{C}_{7}$-cyclitol intermediates by downstream biosynthetic steps has become a major bottleneck during the production of $\mathbf{1}$ (Fig. 1b). To investigate the rate-limiting factors, transcription levels of genes involved in the biosynthesis of $\mathbf{1}$ were surveyed by analyzing the transcriptomes of Actinoplanes sp. SE50/110 collected at 24, 48, or $72 \mathrm{~h}$ during fermentation. The transcription of the phosphoglucomutase gene pgmA (ACPL_2870) and the 4,6-DH gene acbB, critical for the biosynthesis of $\mathbf{6}$, was significantly lower than that of other genes in all three transcriptomes (Supplementary Fig. 45a). Therefore, $p g m A$ and $a c b B$ were individually overexpressed under the control of the promoter kasOp* in Actinoplanes sp. SE50/110, resulting in improved transcription of the genes by 89.2- and 2.7fold, respectively (Fig. 6d; Supplementary Fig. 45b-d). This increase in transcription of pgmA and $a c b B$ directly related to $8.1 \%$ and $9.7 \%$ titer increases of 1 (Fig. 6e), $16.0 \%$ and $15.0 \%$ productivity increases of $\mathbf{1}$ (Supplementary Fig. 46a), 15.1\% and $22.3 \%$ accumulation decreases of 8 , and $9.4 \%$ and $20.4 \%$ accumulation decreases of 9 (Fig. 6f), respectively, when compared with that of the control strain SE50/110::pSET152. Meanwhile, overexpression of these genes did not show any significant effects on biomass accumulation (Supplementary Fig. 46b). These results suggested that insufficient phosphoglucomutase and 4,6-DH activities are among the limiting factors in the production of $\mathbf{1}$.

Since the 2.7-fold improvement of $a c b B$ transcription was far from ideal, multiple copies of $a c b B\left(A B_{n}\right)$ were co-overexpressed with $\operatorname{pgmA}(A P)$ in Actinoplanes sp. SE50/110 (Fig. 6d). The mutant carrying a cassette of $A P-A B_{2}$ had 4.4- and 2.5-fold increases in $a c b B$ transcription and 4,6-DH activity, respectively, and showed the highest titer and productivity improvement of $\mathbf{1}$ (23.4\% and $29.1 \%$, respectively) (Fig. 6e; Supplementary Fig. 46a, $c, d)$. The cassette also caused decreases in the accumulations of $\mathbf{8}$ (21.3\%) and 9 (27.2\%), decreases in the total amounts of endogenous 10 and $11(10.4 \%)$, without any obvious changes in biomass, when compared with that of SE50/110::pSET152 (Fig. 6f; Supplementary Fig. 46b, e).

To avoid any genetic instability of the multiple copies of $a c b B$, heterologous genes encoding 4,6-DHs with higher enzymatic activity from Mycobacterium smegmatis MC2 155 (MB), Pseudomonas aeruginosa M18 (PB), and Escherichia coli K12 W3110 $(E B)^{28-30}$ were individually co-expressed with $A P$ (Fig. 6d). The introduction of $A P-M B$ cassette resulted in a 1.9fold increase in 4,6-DH activity, the highest titer and productivity improvement of $\mathbf{1}(28.9 \%$ and $33.5 \%$, respectively), decreases of $\mathbf{8}$ (25.6\%) and 9 (18.8\%) accumulations, decreases of the total amounts of endogenous 10 and 11 (23.4\%), without any obvious changes in biomass, when compared with that of SE50/110:: pSET152 (Fig. 6e, f; Supplementary Fig. 46a, d-g).

To further enhance the competing utilization of precursor $\mathbf{5}$ for the biosynthesis of 6, cognate G-1-PT genes ${ }^{31-33}$ were individually co-expressed with $A P-M B$ in Actinoplanes sp. SE50/110 (Fig. 6d). The introduction of the AP-MB-EA cassette resulted in a 3.5-fold increase in G-1-PT activity, substantial titer and 
a

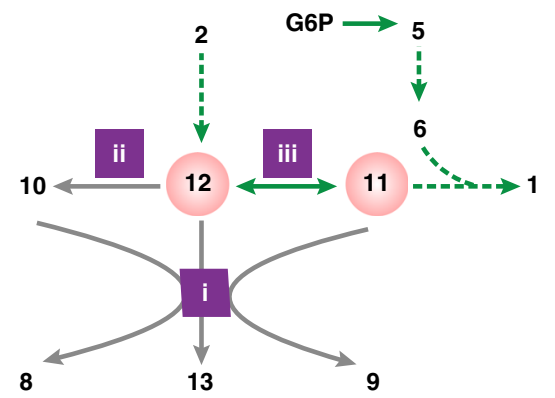

i $a c b J$ ACPL_8310 *

ii $A C P L_{-} 7966$ * $A C P L \_2834$

iii $a c b N$ 个 $A C P L_{-} 7511$ * ACPL_5736 $\mathrm{X}$ ACPL_7667 *

$\vdots$ - Acarbose biosynthetic pathway

- Shunt pathway

Metabolic branch point

Metabolic engineering strategy

$\checkmark$ Down-regulation \& Deletion

Overexpression

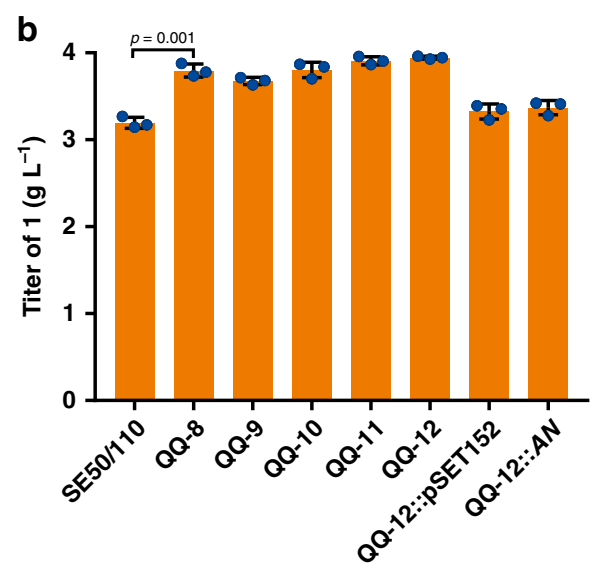

d

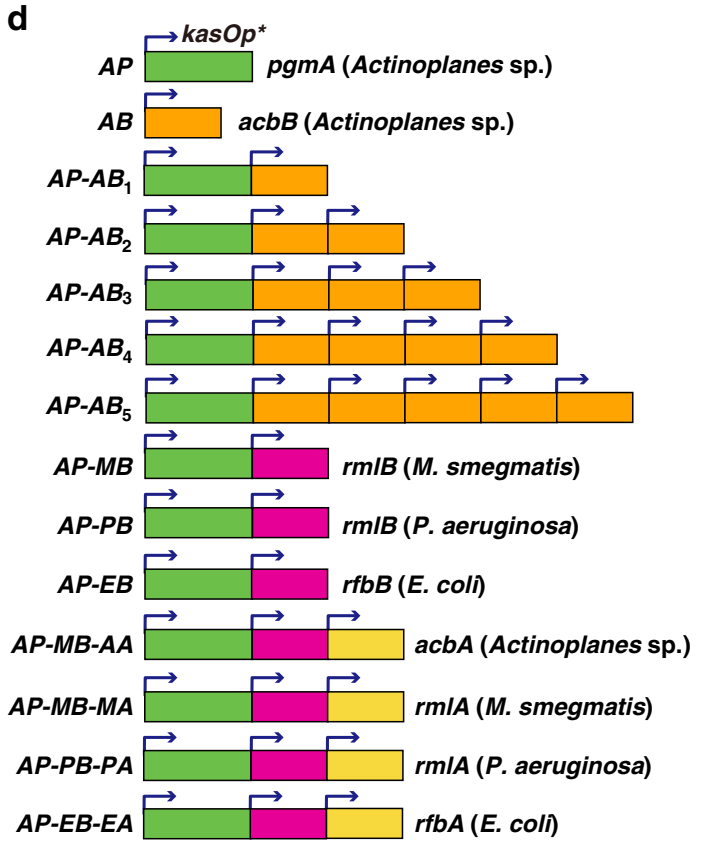

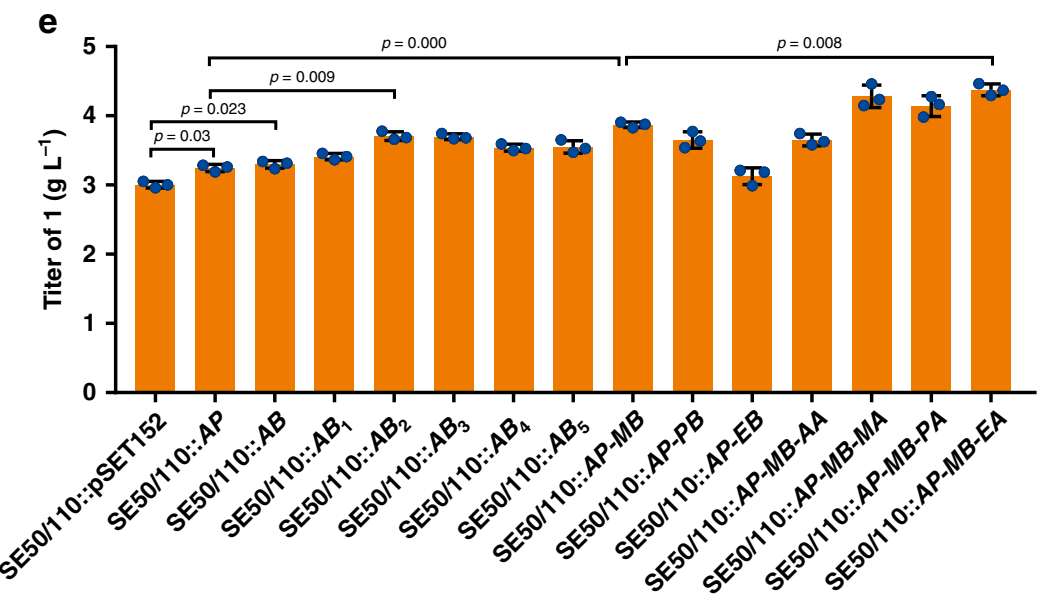
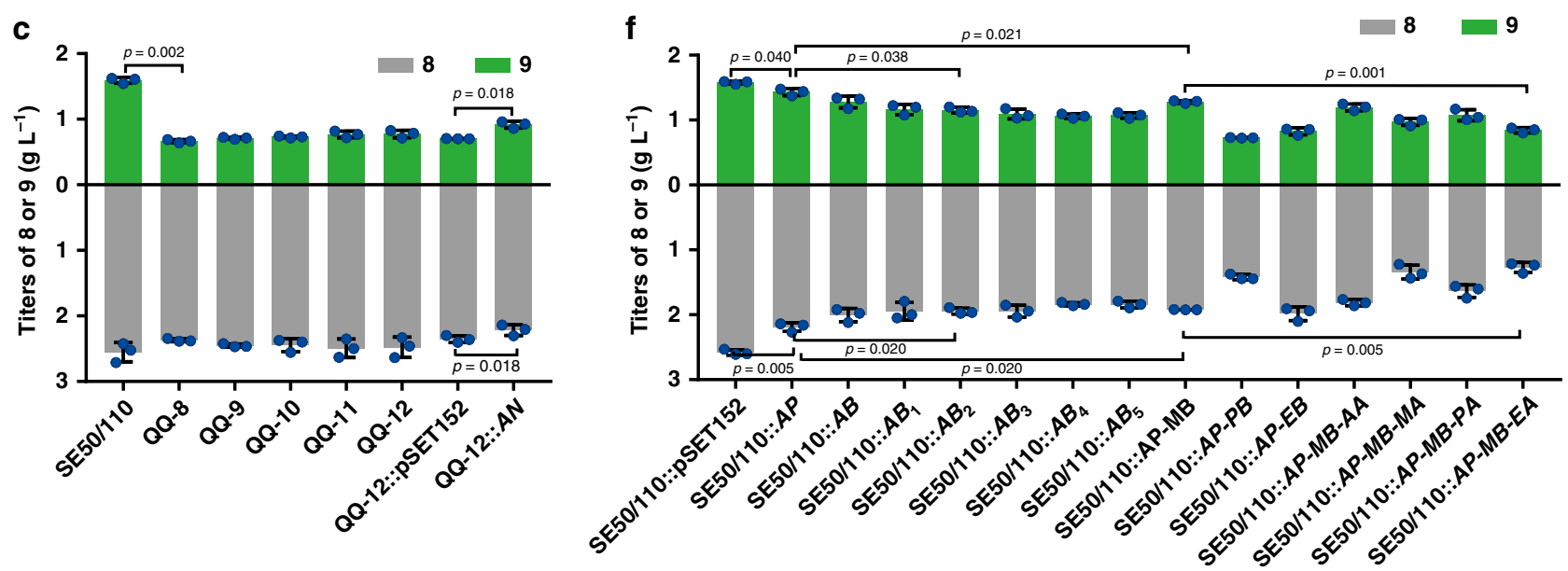

productivity increases of $\mathbf{1}(45.7 \%$ and $56.9 \%$, respectively), decreases in the accumulations of $8(50.6 \%)$ and $9(46.5 \%)$, and decreases in the total amounts of endogenous 10 and $\mathbf{1 1}(35.2 \%)$, and a 6.9\% decrease in biomass, when compared with SE50/110:: pSET152 (Fig. 6e, f; Supplementary Fig. 46a, e-h).
Integration of the effective engineering strategies. For a final combination of these effective metabolic engineering strategies, the cassettes $A P-M B-E A$ and $A P-M B-E A-A N$ were individually introduced into the mutant QQ-12. The expression of $A P-M B-E A$ improved the titer of 1 by $64.8 \%$, which was further increased by 
Fig. 6 Combined metablic engineering strategies for minimizing the flux toward the shunt products and maximizing the supply of the aminodeoxyhexose moiety. a Schematic illustration of the strategies for diverting the metabolic flux of shunt products toward 1. b, c The titers of 1, 8, and $\mathbf{9}$ of Actinoplanes sp. SE50/110 (abbreviated as SE50/110), QQ-8 (down-regulation of the acbJ expression by introducing kasOp*-acbJ in the chromosome of QQ-7), QQ-9 (deletion of ACPL_7966 in QQ-8), QQ-10 (deletion of ACPL_7511 in QQ-9), QQ-11 (deletion of ACPL_5736 in QQ-10), QQ-12 (deletion of ACPL_7667 in QQ-11), QQ-12::pSET152 (as control), and QQ-12::AN (overexpression of $a c b N$ in QQ-12) after fermentation for 4 days. For more details, see also Supplementary Figs. 42-44. d Schematic illustration of increasing the biosynthetic capacity of amino-deoxyhexose moiety by stepwise optimization of biosynthetic reactions at gene dosage or enzymatic activity. e, f The titers of $\mathbf{1}, \mathbf{8}$, and $\mathbf{9}$ of mutants carrying pSET152 (as control) and its derived plasmids containing cassettes $A P, A B, A P-A B_{1}, A P-A B_{2}, A P-A B_{3}, A P-A B_{4}, A P-A B_{5}, A P-M B, A P-P B, A P-E B, A P-M B-A A, A P-M B-M A, A P-M B-P A$, or AP-MB-EA after fermentation for 4 days. Two-tailed paired $t$ tests. Error bars, mean $\pm S D$ ( $n=3$ biological replicates). For more details, see also Supplementary Figs. 45 , 46. Source data underlying Figs. $6 b, c, e, f$ are provided as a Source Data file.

a
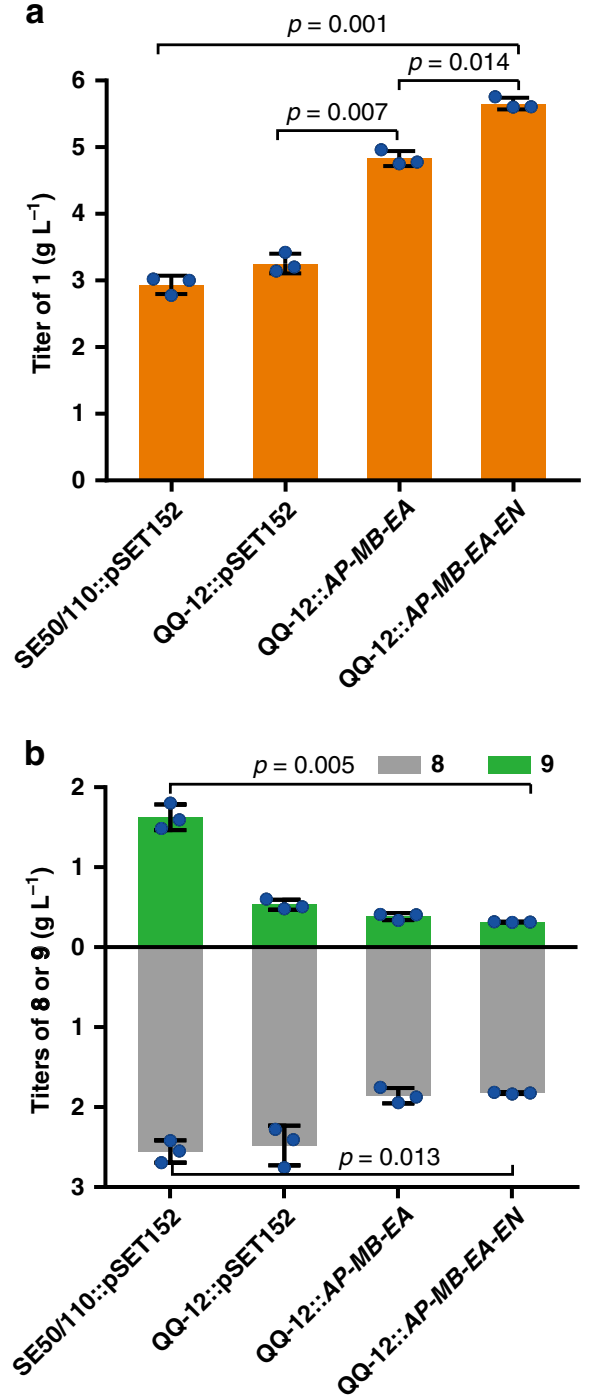

C

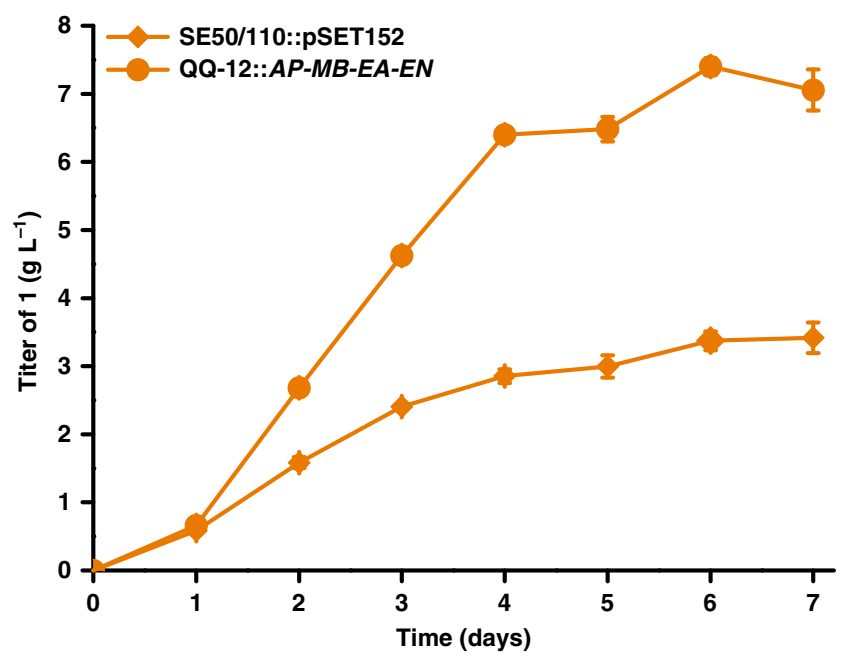

d

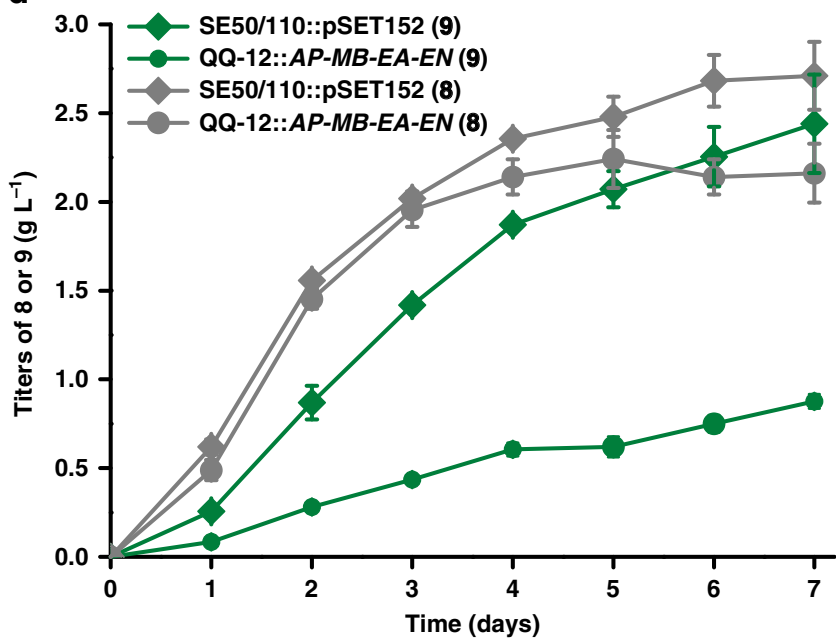

Fig. 7 Integration of the effective engineering strategies. a, b The titers of $\mathbf{1}, \mathbf{8}$, and $\mathbf{9}$ of SE50/110::pSET152 (as a control), QQ-12::pSET152 (as a control), and QQ-12::AP-MB-EA and QQ-12::AP-MB-EA-AN after fermentation for 4 days. Two-tailed paired $t$ tests. c, $\mathbf{d}$ Fed-batch fermentation of SE50/110:: pSET152 (rhombus) and QQ-12::AP-MB-EA-AN (circle). Time courses of $\mathbf{1}$ (orange), 8 (gray), and $\mathbf{9}$ (green) titers were monitored. Error bars, mean \pm SD $(n=3$ biological replicates). For more details, see also Supplementary Fig. 47. Source data are provided as a Source Data file.

$17.0 \%$ when $A N$ was introduced (Fig. 7a, b; Supplementary Fig. 47). The best engineered strain, QQ-12::AP-MB-EA-AN, produced $7.4 \mathrm{~g} \mathrm{~L}^{-1}$ of $\mathbf{1}$ in 6-day fed-batch fermentation, representing a 1.2 -fold titer and 2.3 -fold productivity improvement of 1, a $20.2 \%$ decrease of $\mathbf{8}$, and a $67.1 \%$ decrease of $\mathbf{9}$ (Fig. 7c, d). However, an unexpected $33.0 \%$ decrease in biomass occurred when compared with the control strain SE50/110::pSET152 (Supplementary Fig. 47c).

\section{Discussion}

Acarbose (1) is one of the most widely used anti-diabetic drugs, yet its biosynthesis remained somewhat elusive despite a decadeslong clinical application 1 . While previous studies on 1 biosynthesis have revealed the biosynthetic steps from 2 to $\mathbf{4}^{20,21}$, further downstream, the pathway has yet to be biochemically established. Recently, the formation of a cyclitol moiety in pyralomicin has been proposed to involve dehydration of 4 to 12 and 
then reduction of $\mathbf{1 2}$ to $\mathbf{1 1}$. However, the functions of the proposed enzymes, PrlW (cyclitol oxidoreductase) and PrlV (cyclitol dehydrogenase), have not been experimentally characterized ${ }^{34}$. In the present study, clarification of the functions of AcbL and AcbN through metabolic analysis, chemical incorporation, and biochemical experiments not only made a breakthrough in the study of 1 biosynthesis, but also contributed to a better understanding of the biosynthesis of other $\mathrm{C}_{7} \mathrm{~N}$-aminocyclitols ${ }^{34-36}$.

Accordingly, the subsequent biosynthetic steps of $\mathrm{C}_{7}$-cyclitol moiety proposed in the previous study ${ }^{1}$ was updated with phosphorylation of $\mathbf{1 1}$ to valienol-1,7-diP and nucleotidylation of the product to NDP-valienol-7-P (Fig. 1b). Although AcbJ was demonstrated to be essential for the biosynthesis of $\mathbf{1}$ through gene deletion in Actinoplanes sp. SE50/110 (Supplementary Fig. 17), its definite function is still unclear. However, since AcbJ can catalyze the dephosphorylation of $\mathbf{1 1}$, we speculate that it is responsible for the hydrolysis of the $\mathrm{C}-7$ phosphate group of the proposed intermediates valienol-1,7-diP, NDP-valienol-7-P, or dTDP-acarviose-7-P (Fig. 1b). This will be a subject of our future investigation related to $\mathbf{1}$ biosynthesis.

In line with the results of previous feeding studies with ${ }^{13} \mathrm{C}$ labeled 8 and 13, in which none of these compounds were incorporated into $\mathbf{1}^{19}$, our work showed that both compounds are shunt products branched from the biosynthetic intermediate 12 (Fig. 1b; Supplementary Fig. 33). Although 8 could be converted to 10 by kinase(s) in the CFE of Actinoplanes sp. SE50/11037, the reaction does not seem to be part of $\mathbf{1}$ biosynthesis or a salvage pathway to $\mathbf{1}$, as our study showed that $\mathbf{1 0}$ is also a shunt product directly derived from 12 (Figs. $1 \mathrm{~b}$ and 5). It has also been reported that the CFE of Actinoplanes sp. SE50/110 showed weak kinase activity toward $\mathbf{9}$ and presumably generated a low amount of $\mathbf{1 1}{ }^{37}$. However, the production of $\mathbf{1}$ was not detected after feeding QQ-4 with 9 in our study, except when the $\mathrm{C}_{7}$-cyclitol kinase gene valC was introduced in the chromosome as in QQ-4::valC (Fig. 2c; Supplementary Fig. 20), suggesting that the weak kinase activity might be insufficient to produce detectable 1 .

The identified shunt products $\mathbf{8 , 9}, \mathbf{1 0}$, and $\mathbf{1 3}$ are branched from the metabolic intermediates 11 and $\mathbf{1 2}$ (Fig. 1b). The substrate promiscuities of the involved phosphatases and oxidoreductases control the branch points and thereby establish complicated crosstalks between the shunt pathways, $\mathbf{1}$ biosynthesis, and primary metabolism. In this network, AcbJ is a crucial switch that controls the metabolic flux toward $\mathbf{1}$ and shunt products. Likewise, the putative hydrolase (ACPL_8310), $\beta$-phosphoglucomutase (ACPL_2834), trehalose 6-phosphate phosphatase (ACPL_7709), gfo/Idh/MocA family oxidoreductase (ACPL_7966), inositol 2-dehydrogenase (ACPL_7511), glucose-6-phosphate 1-dehydrogenase (ACPL_5736), and acylCoA dehydrogenase (ACPL_7667) play important roles in primary metabolism and contribute to the biosynthesis of the shunt products as well. Accordingly, the similar shunt pathways were also speculated to exist in the biosynthetic pathways of other $\mathrm{C}_{7} \mathrm{~N}$-aminocyclitols ${ }^{34,35}$

The high intracellular levels of phosphorylated metabolites are usually toxic and cause growth inhibition ${ }^{38,39}$. This is supported by the fact that deletion of $a c b J$ resulted in a significant increase in intracellular phosphorylated $\mathrm{C}_{7}$-cyclitols (10 and 11) and obvious decrease in the biomass compared with that of Actinoplanes sp. SE50/110 (Supplementary Fig. 17). Meanwhile, the growth inhibition was gradually alleviated along with the increase in phosphatase activities and the decrease in total amounts of endogenous $\mathbf{1 0}$ and $\mathbf{1 1}$ due to the trans-complementation of $a c b J$ under the control of promoters with different strengths (Supplementary Fig. 42). These evidences suggest that dephosphorylation of accumulated phosphorylated $\mathrm{C}_{7}$-cyclitols (10 and 11) by the phosphatases and efflux of the dephosphorylated products (8 and 9) are parts of a detoxification process in cells. However, it is still unclear why the deletion of $a c b J$ resulted in a substantial decrease in the accumulation of 9 , but had no obvious effect on that of 8 (Supplementary Fig. 17c). We proposed that the accumulated intermediate $\mathbf{1 1}$, caused by the blockage of $\mathbf{1}$ biosynthesis and low dephosphorylation activity in the acbJ deletion mutant, is reversely oxidized to $\mathbf{1 2}$ by AcbN and then reduced to $\mathbf{1 0}$ by the reductases (Fig. 1b). This may contribute to the relatively constant concentration of $\mathbf{8}$. However, this hypothesis needs to be further confirmed through quantification of intracellular $\mathbf{1 0}$ and $\mathbf{1 1}$.

We found that an imbalanced metabolic flux caused by the insufficient biosynthesis of amino-deoxyhexose moiety is another crucial factor for the leakage of $\mathrm{C}_{7}$-cyclitol intermediates. Therefore, employment of multiple strategies to reduce the metabolic flux to shunt products resulted in only a moderate increase of $\mathbf{1}$, but caused a substantial increase in the intracellular concentrations of phosphorylated $\mathrm{C}_{7}$-cyclitols (Fig. 6a, b; Supplementary Fig. 44c). This agrees well with the result of a recent study, in which overexpression of $a c b C$ in Actinoplanes sp. SE50/110 led to an increase in intracellular 11, rather than the production of $\mathbf{1}^{40}$.

Moreover, the low transcription of $a c b B$ seemed to be a general phenomenon, which was also found in the transcriptome analysis of Actinoplanes sp. SE50/110 cultured in different fermentation media ${ }^{9}$. In our work, two synthetic biology strategies were performed to adjust the activity of 4,6-DH: (1) balancing the transcription levels of $a c b B$ and other genes in the $a c b$ cluster by titrating gene copy numbers; (2) improving the 4,6-DH activity by recruiting heterologous enzymes with higher catalytic activities from M. smegmatis, E. coli, or $P$. aeruginosa ${ }^{28-30}$. Both strategies resulted in successful improvement of the biosynthetic capacity of amino-deoxyhexose moiety (Fig. 6e; Supplementary Fig. 46). The substantial titer improvement of $\mathbf{1}$ with highly efficient heterologous G-1-PTs ${ }^{31-33}$ further demonstrated that overexpression of enzymes that divert primary metabolites into secondary metabolism is a useful strategy 41 (Fig. 6e). Therefore, these results demonstrated the potential of optimization of the biosynthetic pathway by employing various highly efficient heterogenous isoenzymes.

However, due to the promiscuous activities of the already identified and even more unidentified hydrolases and oxidoreductases $^{38,42-45}$ involved in the accumulation of shunt products, $\sim 58.4 \%$ shunt products (12.2 $\mathrm{mM} 8$ and $4.3 \mathrm{mM} \mathrm{9)}$ still remained in the fermentation broth of the best engineered strain. Meanwhile, the $11.6 \mathrm{mM}$ decreased accumulations of shunt products just resulted in a $6.2 \mathrm{mM}$ increase of 1 titer (Fig. 7c, d), suggesting that the other $5.4 \mathrm{mM} \mathrm{C}_{7}$-cyclitol intermediates remain in the cell or are utilized by other unknown competing metabolic pathways (Supplementary Fig. 47d). These results implied the potential in maximizing the utilization of the $\mathrm{C}_{7^{-}}$ cyclitol intermediates for the titer improvement of $\mathbf{1}$, with a better understanding of biosynthesis and expanded genetic toolbox for rational engineering ${ }^{46,47}$. In addition, the titer of 1 of the best engineered strain is promising for further improvement using optimized fermentation strategies ${ }^{48-50}$.

Taken together, systematic metabolic engineering strategies, minimizing the metabolic flux towards shunt products and maximizing the supply of amino-deoxyhexose moiety to balance the flux with $\mathrm{C}_{7}$-cyclitol moiety, were employed to promote the effective utilization of $\mathrm{C}_{7}$-cyclitol intermediates for the production of 1. Our effort at uncovering and fine-tuning the biosynthetic network of $\mathbf{1}$ ensures an opportunity on understanding the biosynthesis of other $\mathrm{C}_{7} \mathrm{~N}$-aminocyclitols, diagnosing metabolic bottlenecks and enhancing yields through pathway engineering $17,24,34,35,51$. 


\section{Methods}

Strains and their culture conditions. Strains, plasmids, and primers used in this study are listed in Supplementary Table 8, Supplementary Table 9, and Supplementary Data 1, respectively.

Escherichia coli DH10B was used for gene cloning, ET12567(pUZ8002) was used for the intergeneric conjugation between E. coli and mycelia of Actinoplanes sp., and BL21(DE3) was used for the heterologous expression of proteins. Escherichia coli were cultured with Luria-Bertani (LB) broth or on LB agar plates at $37^{\circ} \mathrm{C}$. Actinoplanes sp. SE50/110 and its derivatives were cultivated on STY agar medium (sucrose $3 \%$, tryptone $0.5 \%$, yeast extract $0.5 \%$, casin hydrolysate $0.1 \%$, $\mathrm{K}_{2} \mathrm{HPO}_{4} \cdot 3 \mathrm{H}_{2} \mathrm{O} 0.1 \%$, $\mathrm{KCl} 0.05 \%, \mathrm{FeSO}_{4} 0.005 \%$, agar $2 \%, \mathrm{pH} 7.2$ ) at $30^{\circ} \mathrm{C}$ for 2-3 days, and then inoculated into $30 \mathrm{~mL}$ of SM broth (glucose $1.5 \%$, maltose $1 \%$, $\mathrm{K}_{2} \mathrm{HPO}_{4} \cdot 3 \mathrm{H}_{2} \mathrm{O} 0.1 \%$, glycerol $1 \%$, malt extract $1 \%$, tryptone $0.5 \%$, yeast extract $0.5 \%$, casin hydrolysate $0.1 \%, \mathrm{pH} 7.2$ ) in $250 \mathrm{~mL}$ baffled flasks for about $36 \mathrm{~h}$ on rotary shaker $\left(30^{\circ} \mathrm{C}, 220\right.$ r.p.m.). The medium for the seed culture contains $1.5 \%$ maltose, $1 \%$ glucose, $4 \%$ soya flour, $1 \%$ glycerol, $1 \%$ soluble starch, and $0.2 \%$ $\mathrm{CaCO}_{3}, \mathrm{pH}$ 7.2. The fermentation medium contains $5 \%$ maltose, $3 \%$ glucose, $1 \%$ soya flour, $0.3 \%$ glutamate, $0.3 \%$ yeast (Angel Yeast Co., Ltd), $0.1 \% \mathrm{~K}_{2} \mathrm{HPO}_{4} \cdot 3 \mathrm{H}_{2} \mathrm{O}$, $0.05 \% \mathrm{FeCl}_{3}$, and $0.25 \% \mathrm{CaCO}_{3}, \mathrm{pH} 7.2$. When needed, antibiotics were added to final concentrations of $50 \mathrm{mg} \mathrm{L}^{-1}$ for apramycin and kanamycin, and $25 \mathrm{mg} \mathrm{L}^{-1}$ for chloramphenicol.

To assess the production of $\mathbf{1}$ and biomass of Actinoplanes sp. SE50/110 and its derivatives, $4-\mathrm{mL}$ of SM culture was transferred to $40 \mathrm{~mL}$ of seed medium in $250 \mathrm{~mL}$ baffled flask and cultivated for $28-30 \mathrm{~h}$ on rotary shaker $\left(30^{\circ} \mathrm{C}, 220\right.$ r.p.m.). Then, $7.5-\mathrm{mL}$ seed culture was inoculated to $50 \mathrm{~mL}$ of fermentation medium in $250 \mathrm{~mL}$ baffled flask and cultivated for another 4 days. Additionally, 1 g glucose was added to each flask on the second day. Then, the production of $\mathbf{1}$ and the biomass (dry cell weight) was analyzed after 4-day fermentation.

To assess the potential of the engineered strains, the fed-batch fermentation was conducted in baffled flasks using feeding strategy that $0.5 \mathrm{~g}$ glucose was added at $24 \mathrm{~h}$, and then $0.75 \mathrm{~g}$ glucose and $0.125 \mathrm{~g}$ maltose were added every $24 \mathrm{~h}$. The fermentation was continued for 7 days on a rotary shaker $\left(30^{\circ} \mathrm{C}, 220\right.$ r.p.m.).

The involved chemicals and their sources. Synthesis of oligonucleotide primers and sequencing of PCR products were performed by Tsingke Biological Technology. 5-Flucytosine was purchased from Adamas. The derivatization reagent BSTFA:TMCS (99:1) was purchased from Sigma-Aldrich. The standards of valienol (9) (Supplementary Fig. 48a) and valienone (13) were synthesized by WuXi Apptec Co., Ltd. The standard of 1-epi-valienol (8) was purified from the fermentation broth of SE50/110 $\triangle a c b N$ and confirmed by NMR spectroscopy (Supplementary Fig. 48b). The standards of dTDP-D-glucose and dTDP-4-keto-6-deoxy-D-glucose were purchased from Carbosynth China Ltd. The molecular biology reagents were purchased from Thermo Fisher Scientific and Takara. Other biochemical reagents were purchased from Sinopharm Chemical Reagent Co., Ltd., Oxoid, Sangon Biotech, and Sigma-Aldrich.

Manipulation of genes. For the deletion of the $a c b$ cluster (containing 32.2-kb region of DNA from $a c b Z$ to the upstream region of $a c b D$ ), homologous flanking sequences ( $a c b$ - $\mathrm{L}$ and $a c b$-R) were amplified with corresponding primers (Supplementary Data 1) from the genome of Actinoplanes sp. SE50/110 (GenBank accession no. CP003170.1) and cloned into the Actinomycete-E. coli shuttle vector pLQ752 to give pLQ759, which was further verified by sequencing. Plasmids pLQ760, pLQ761, pLQ762, pLQ765, pLQ766, pLQ767, pLQ768, and pLQ769 were similarly constructed for the in-frame deletions of $a c b C$, acbJ, ACPL 8310 , $A C P L \_7966, A C P L \_7511, A C P L \_5736, A C P L \_7667$, and $a c b N$, respectively.

To reduce the transcription of $A C P L \_2834$, two homologous flanking sequences (2834d-L and 2834d-R) and $W V$ promoter $(W V p)$ (Supplementary Data 2) of the $a c b$ cluster were amplified from the genome of Actinoplanes sp. SE50/110. The $W V p$ and $2834 \mathrm{~d}-\mathrm{R}$ were ligated by overlapping PCR and cloned into pLQ752 together with $2834 \mathrm{~d}-\mathrm{L}$ to generate pLQ763. For reducing the $a c b J$ transcription, two homologous flanking sequences ( $a c b J d-\mathrm{L}$ and $a c b J d-\mathrm{R})$ were amplified from the genome of Actinoplanes sp. SE50/110 and cloned into pLQ752. Subsequently, the $k a s O p^{*}-a c b J$ was amplified from pSET152-kasOp*-acbJ and inserted between these two homologous flanking sequences to generate pLQ764.

The recombinant plasmids were transferred to ET12567(pUZ8002) and then introduced into Actinoplanes sp. SE50/110 by intergeneric conjugation. The methods of intergeneric conjugation, and mutant construction and selection were described in the Supplementary Method. The schematic representation of gene deletions and insertions in the chromosome and the verification of the mutants by PCR were shown in Supplementary Figs. 49-54. Meanwhile, the corresponding PCR products were further verified by DNA sequencing.

Overexpression or complementation of genes. For the expression of single gene, promoters $k a s O p^{*}$ and $e r m E p^{*}$, and endogenous promoters gapAp, groLp, and $W V p$ were, respectively, amplified from pDR-4-K*, pIB139, and the genome of Actinoplanes sp. SE50/110 with corresponding primers (Supplementary Data $1-4)^{52-54}$ Meanwhile, gene valC was amplified from the genome of $S$. hygroscopicus var. jinggangensis 5008 (GenBank accession no. CP003275.1) ${ }^{25}$, and genes $a c b J$, acbN, $a c b A, a c b B$, and $\operatorname{pgm} A$ were amplified from the genome of Actinoplanes sp. SE50/110.
The corresponding promoters and genes were ligated by overlapping PCR, cloned into pSET152 55 , and verified by sequencing.

For the construction of pSET152-AP-AB $B_{n}$, the kasOp*-pgmA $(A P)$ was amplified using primers $k a s O p^{*}-X b a \mathrm{I}-\mathrm{F} / p g m A-A f l \mathrm{II}-\mathrm{R}$ and then cloned into the pSET152-M to generate pSET152-AP. Subsequently, fragments of $k a s O p^{*}-a c b B$ $(A B)$ with different flanking restriction sites of $A f l \mathrm{II} / B g l \mathrm{II}, B g l \mathrm{II} / K p n \mathrm{I}, K p n \mathrm{I} / N d e \mathrm{I}$, $\mathrm{NdeI} / \mathrm{ScaI}$, or ScaI/MfeI were individually amplified using corresponding primers (Supplementary Data 1 ) and successively cloned into pSET152-AP to generate pSET152- $A P-A B_{1}$, pSET152- $A P-A B_{2}$, pSET152- $A P-A B_{3}$, pSET152- $A P-A B_{4}$, or pSET152- $A P-A B_{5}$.

For the co-expression of the heterogenous genes encoding 4,6-DHs, they were, respectively, amplified from the genomes of $M$. smegmatis MC2 155 ( $M B$, GenBank accession no. CP000480.1), P. aeruginosa M18 (PB, GenBank accession no. CP002496.1), and E. coli K12 W3110 (EB, GenBank accession no. AP009048.1) using corresponding primers, ligated with the $k a s O p^{*}$ promoter by overlapping PCR, and then cloned into pSET152- $A P$ to generate pSET152- $A P-M B$, pSET152 $A P-P B$, and pSET152- $A P-E B$. Subsequently, cognate G-1-PT genes $A A, M A, P A$, and $E A$ were obtained in a similar way and further inserted into pSET152-AP-MB to generate pSET152- $A P-M B-A A$, pSET152- $A P-M B-M A$, pSET152-AP-MB-PA, and pSET152- $A P-M B-E A$, respectively.

The recombinant plasmids were transferred to ET12567(pUZ8002) and then introduced into Actinoplanes sp. SE50/110 and the corresponding derivatives by intergeneric conjugation. The exconjugants were selected, cultured, verified by PCR, and the corresponding PCR products were further verified by DNA sequencing (Supplementary Method 1)

Purification and identification of compounds in P-1. For the preparation of compounds in P-1 (Fig. 2a), $1 \mathrm{~L}$ of fermentation broth of Actinoplanes sp. SE50/110 was harvested by centrifugation. The supernatant was adjusted to $\mathrm{pH} 3.0-4.0$ with oxalic acid. After removing the precipitate by centrifugation, the supernatant was adjusted with ammonium hydroxide to $\mathrm{pH} 7.0$ and mixed with anion exchange resin D201 ( $1 \mathrm{~g}$ for $10 \mathrm{~mL}$ broth) for about $1 \mathrm{~h}$ to remove pigments. Then, the mixture was filtered through Whatman filter paper and concentrated to $200 \mathrm{~mL}$ using vacuum evaporator at $40^{\circ} \mathrm{C}$. About 10 -mL concentrated liquor was subjected to column filled with pre-treated aminopropyl-functionalized silica gel each time, and eluted with acetonitrile/water $(70: 30, \mathrm{v} / \mathrm{v})$ to many fractions. As monitored by HPLC, fractions containing the target compounds were pooled, concentrated, and freeze-dried.

For subsequent purification, the powder was dissolved in 5-mL Milli-Q water and then subjected to Sephadex LH-20 $(2 \times 200 \mathrm{~cm})$ column twice. The column was eluted with methanol/water $(30: 70, \mathrm{v} / \mathrm{v})$, and fractions containing the target compounds were pooled. After concentration, the sample was separated again on Sephadex LH-20 column, and maltose was removed. For the removal of glucose residue, the sample was dissolved in water and treated with hexokinase at $30^{\circ} \mathrm{C}$ for $1 \mathrm{~h}$. The reaction product was freeze-dried, dissolved in 2-3 $\mathrm{mL}$ of Milli-Q water, and separated twice on Sephadex LH-20 column. Further separation was performed on analytical HPLC (Agilent series 1260, Agilent Technologies, USA) with Agilent ZORBAX SB-Aq $\left(4.6 \times 250 \mathrm{~mm}^{2}\right.$, particle size $\left.5 \mu \mathrm{m}\right)$ at a flow rate of $1 \mathrm{~mL} \mathrm{~min}^{-1}$ using an elution buffer of water/acetonitrile $(99.5: 0.5, \mathrm{v} / \mathrm{v})$. The purified compounds were freeze-dried.

Purified compounds were dissolved in $\mathrm{D}_{2} \mathrm{O}$, and the one-dimensional (1D) $\left({ }^{1} \mathrm{H}\right.$, ${ }^{13} \mathrm{C}$, and distortionless enhancement by polarization transfer) and $2 \mathrm{D}\left({ }^{1} \mathrm{H}-{ }^{1} \mathrm{H}\right.$ correlation spectroscopy [COSY], heteronuclear single-quantum correlation spectroscopy [HSQC], hetero-nuclear multiple-bond correlation spectroscopy [HMBC], and nuclear overhauser effect [NOESY]) NMR spectra were collected in $\mathrm{D}_{2} \mathrm{O}$ at $600 \mathrm{MHz}\left({ }^{1} \mathrm{H} \mathrm{NMR}\right)$ and $150 \mathrm{MHz}\left({ }^{13} \mathrm{C} \mathrm{NMR}\right)$ on Bruker Avance III 600 spectrometer (magnetic field strength with $14.09 \mathrm{~T}$ ). The NMR data processing was performed using the software of MestReNova 9.0.1.

Expression and purification of proteins. For the expression of $\mathrm{ValC}$, gene valC was amplified from the genome of S. hygroscopicus var. jinggangensis 5008 and cloned into pET-30a ${ }^{56}$ to generate pET30a-valC. BL21(DE3) with the recombinant plasmid was cultured in LB medium containing kanamycin $(50 \mu \mathrm{g} / \mathrm{mL})$ at $37^{\circ} \mathrm{C}$ to $\mathrm{OD}_{600 \mathrm{~nm}}$ of $0.6-0.8$. Protein expression was induced with IPTG (isopropyl $\beta$-D-1thiogalactopyranosid) $(0.4 \mathrm{mM})$ at $16^{\circ} \mathrm{C}$ for $12-16 \mathrm{~h}$. Cells were harvested by centrifugation, re-suspended in buffer TN $(25 \mathrm{mM}$ Tris- $\mathrm{HCl}, \mathrm{pH} 8.0,300 \mathrm{mM}$ $\mathrm{NaCl}$ ), and lysed by sonication. The supernatant was separated from the cell debris by centrifugation at $15,777 \times g$ for $45 \mathrm{~min}$ at $4^{\circ} \mathrm{C}$, and passed through pre-treated nickel-affinity chromatography. After pre-wash of the column with $50-\mathrm{mL}$ washing buffer (25 mM Tris-HCl, pH 8.0, $300 \mathrm{mM} \mathrm{NaCl}, 25 \mathrm{mM}$ imidazole), the target protein was eluted with elution buffer $(25 \mathrm{mM}$ Tris- $\mathrm{HCl}, \mathrm{pH} 8.0,300 \mathrm{mM} \mathrm{NaCl}$, $250 \mathrm{mM}$ imidazole). The purified protein was concentrated and exchanged with buffer TN using $10 \mathrm{kDa}$ concentrator (Millipore Amicon Ultra), and checked by sodium dodecyl sulfate-polyacrylamide gel electrophoresis, and its concentration was determined by Bradford Protein Assay Kit (Sangon Biotech). The protein was used for enzymatic assay or stored in $10 \%$ glycerol at $-80^{\circ} \mathrm{C}$.

The pET30a- $a c b M$, pET30a- $a c b N$, pET30a- $a c b O$, and pET30a- $a c b L$ were expressed in BL21(DE3)/pGro7, which were cultured in LB supplemented with kanamycin $\left(50 \mathrm{mg} \mathrm{L}^{-1}\right)$, chloramphenicol $\left(25 \mathrm{mg} \mathrm{L}^{-1}\right)$, and L-arabinose $\left(2 \mathrm{~g} \mathrm{~L}^{-1}\right)$ 
in the beginning. Additionally, the procedures of expression and purification of these proteins were similar with that for ValC.

Preparation and characterization of phosphorylated products. For the preparation of 10, a $5-\mathrm{mL}$ reaction mixture containing $25 \mathrm{mM}$ Tris- $\mathrm{HCl}(\mathrm{pH} 7.5)$, $10 \mathrm{mM} \mathrm{MgCl}_{2}, 10 \mathrm{mM} \mathrm{NH}_{4} \mathrm{Cl}, 10 \mathrm{mM} \mathrm{ATP}, 4.5 \mathrm{mM} \mathrm{8}$, and $6 \mu \mathrm{M} \mathrm{ValC}$ was incubated at $30^{\circ} \mathrm{C}$ for $6 \mathrm{~h}$. The reaction was stopped by adding two volumes of methanol followed with vigorous vortex to denature the proteins. The mixture was centrifuged at $15,777 \times g$ for $10 \mathrm{~min}$, and the supernatant was concentrated and freeze-dried. The powder was dissolved in 1-mL water and subjected to Sephadex LH-20 column. Fractions containing target compound were pooled. The ${ }^{1} \mathrm{H}$ and ${ }^{13} \mathrm{C}$ NMR spectra of the purified compound were collected in $\mathrm{D}_{2} \mathrm{O}$ at $600 / 700 \mathrm{MHz}$ $\left({ }^{1} \mathrm{H}\right.$ NMR) and $150 / 175 \mathrm{MHz}\left({ }^{13} \mathrm{C}\right.$ NMR $)$ on Bruker Avance III 600/AVANCE NEO 700 spectrometers (magnetic field strength with 14.09/16.44 T). The same method was used to prepare, purify, and characterize $\mathbf{1 1}$ and $\mathbf{1 2 .}$

HPLC analysis of the titer of 1. The supernatant of fermentation broth was obtained by centrifugation at $15,777 \times g$ for $10 \mathrm{~min}$ and diluted for 5 folds. The concentration of 1 was analyzed by HPLC (Agilent series 1260, Agilent Technologies, USA) with Agilent ZORBAX NH $5 \mu \mathrm{m})$ using an elution buffer of acetonitrile/phosphate buffer $(65: 35, \mathrm{v} / \mathrm{v})$ at a flow rate of $1 \mathrm{~mL} \mathrm{~min}^{-1}$ and detected at $210 \mathrm{~nm}$. The titer of $\mathbf{1}$ was calculated according to the corresponding standard curves and processed by the GraphPad Prism 7 and Origin 8.1 softwares.

HPLC-TOF/MS analysis of the related compounds. The enzymatic reaction mixtures and related compounds were analyzed by HPLC-time-of-flight/mass spectrometry (HPLC-TOF/MS) (Agilent 1290-6500 Q-TOF) with Agilent ZORBAX SB-Aq $\left(4.6 \times 250 \mathrm{~mm}^{2}\right.$, particle size $\left.5 \mu \mathrm{m}\right)$ at a flow rate of $0.4 \mathrm{~mL} \mathrm{~min}^{-1}$ using an elution buffer composed of (A) Milli-Q water and (B) methanol with the gradient elution procedures: $0-20 \mathrm{~min}, 0.5 \% \mathrm{~B} ; 20-22 \mathrm{~min}, 0.5 \% \mathrm{~B}$ to $95 \% \mathrm{~B} ; 22-27$ $\mathrm{min}, 95 \% \mathrm{~B} ; 27-28 \mathrm{~min}, 95 \% \mathrm{~B}$ to $0.5 \% \mathrm{~B} ; 28-38 \mathrm{~min}, 0.5 \% \mathrm{~B}$. The related compounds were detected in negative ion mode and their calculated $\mathrm{m} / \mathrm{z}$ were listed in Supplementary Table 10.

HPLC-QQQ/MS analysis of the related compounds. Production of $\mathbf{1}$ in feeding experiments, concentrations of endogenous $\mathbf{1 0}$ and $\mathbf{1 1}$, and the products of enzymatic reactions were quantified by HPLC-triple quadrupole/mass spectrometry (HPLC-QQQ/MS) (Agilent 1100 series LC/MSD Trap System), working in the Multiple Reaction Monitoring model by employment of an unique precursor ion-to-product ion for each analyte to achieve highest selectivity and sensitivity. The parameters of polarity, precursor ion, product ion, fragmentor, and collision energy for the related compounds were individually optimized and listed in Supplementary Table 10. HPLC method was the same as that of HPLC-TOF/MS.

GC-QMS analysis of the three shunt products. Twenty-microliter samples of pre-treated reaction mixtures or 50 -fold diluted fermentation broth were dried in vacuum and dissolved in equal volume of pyridine. Ten-microliter solution was mixed with $20 \mu \mathrm{L}$ of derivatization reagent (BSTFA:TMCS $=99: 1$ ) in the vitreous insert and put in a sealed GC vial rapidly, and then incubated at $70^{\circ} \mathrm{C}$ for $45 \mathrm{~min}$. The derived samples were analyzed by GC-QMS (Agilent Technologies, $6850 \mathrm{GC} /$ 5975C MSD) equipped with a capillary column (HP-5 ms; $30 \mathrm{~m} \times 0.25 \mathrm{~mm}^{2}$ ). The oven program was as follows: $70^{\circ} \mathrm{C}$ for $2 \mathrm{~min}$, ramp to $130^{\circ} \mathrm{C}$ at $5^{\circ} \mathrm{C} / \mathrm{min}$, ramp to $180^{\circ} \mathrm{C}$ at $10^{\circ} \mathrm{C} / \mathrm{min}$, ramp to $285^{\circ} \mathrm{C}$ at $5^{\circ} \mathrm{C} / \mathrm{min}$, held for $5 \mathrm{~min}$. The standards of $\mathbf{8}, \mathbf{9}$, and 13 were derivatized to TMS-1-epi-valienol, TMS-valienol, and TMSvalienone, respectively, by BSTFA (TMS is the abbreviation of trimethyl silicyl) and analyzed by GC-QMS. Their mass spectra were showed in Supplementary Table 10, Supplementary Fig. 11, and Supplementary Fig. 23. The same method was used for the quantitative analysis of $\mathbf{8}$ and $\mathbf{9}$ via GC-QQQ/MS (Thermo Scientific, TSQ 8000 ) by tracing their unique product ion $m / z=332.2$. Their titers were calculated according to the corresponding standard curves and processed by the GraphPad Prism 7 and Origin 8.1 softwares.

Analysis of biomass and time courses of cell growth. For analysis of biomass, the mycelia were harvested from $1-\mathrm{mL}$ fermentation culture by centrifugation, washed with $0.25 \mathrm{M}$ hydrochloric acid, and dried at $105^{\circ} \mathrm{C}$ in oven until constant weight reached. The dry cell weight was detected with precision balance. To obtain the time courses of cell growth, $1-\mathrm{mL}$ fermentation culture was sampled every $24 \mathrm{~h}$, and the dry cell weight was measured. The fermentation lasted for 7 days, and $1 \mathrm{~g}$ glucose was added to each flask on days 2 and 4 .

Significance analysis of the amounts of 1 and shunt products. Two-tailed paired $t$ tests were used for the statistical analysis, which was conducted by SPSS (Statistical Product and Service Solutions) software ${ }^{57}$. The software (IBM SPSS Statistics 20) compared the control groups with the corresponding experimental groups and gave $p$ values. $P<0.05, p<0.01$, and $p<0.001$ are defined as significant, moderately significant, and highly significant, respectively.
In vitro enzymatic assays. For the enzymatic assay of $\mathrm{ValC}$, a $20-\mu \mathrm{L}$ reaction mixture, containing $25 \mathrm{mM}$ Tris- $\mathrm{HCl}$ (pH 7.5), $10 \mathrm{mM} \mathrm{MgCl}_{2}, 10 \mathrm{mM} \mathrm{NH}_{4} \mathrm{Cl}$, $10 \mathrm{mM}$ ATP, $2.5 \mathrm{mM} 8$ or 9 , and $6 \mu \mathrm{M}$ enzyme, was incubated at $30^{\circ} \mathrm{C}$ for $1 \mathrm{~h}$. The reaction mixture with boiled ValC was set as control. The reaction was stopped by adding two volumes of methanol, followed by vigorous vortex to denature the proteins. The mixture was centrifuged at $15,777 \times g$ for $20 \mathrm{~min}$, and the supernatant was subjected to HPLC-TOF/MS analysis.

For the enzymatic assay of phosphatase AcbJ, a $20-\mu \mathrm{L}$ reaction mixture, containing $25 \mathrm{mM}$ Tris- $\mathrm{HCl}$ (pH 7.5), $10 \mathrm{mM} \mathrm{MgCl}_{2}, 10 \mathrm{mM} \mathrm{NH}_{4} \mathrm{Cl}, 1.25 \mathrm{mM}$ phosphorylated compounds (10,11, or 12), and $3 \mu \mathrm{M}$ enzyme, was incubated at $30^{\circ} \mathrm{C}$ for 0.5 or $6 \mathrm{~h}$. The reaction mixtures with boiled AcbJ and without enzyme were set as controls. The 6 -h reaction mixture was analyzed by HPLC-TOF/MS and GC-QMS. The concentrations of dephosphorylated compounds $(\mathbf{8}, \mathbf{9}$, or $\mathbf{1 3})$ in 0.5 -h reaction mixture were quantified by GC-QQQ/MS to calculate enzymatic activity. One enzyme unit (U) is defined as the amount of dephosphorylated compound in nanomole produced in $1 \mathrm{~min}$. The same procedure was used to analyze the enzymatic activities of phosphatases ACPL_8310, ACPL_2834, and ACPL 7709 .

For the enzymatic assay of AcbL, substrate 3 was purified from the fermentation broth of TL01 $\Delta$ valD following the separation procedure of $\mathbf{8}$ or $\mathbf{9}$. A total of 2.5 $\mathrm{mM}$ of 3 was added to a reaction mixture, containing $25 \mathrm{mM}$ Tris- $\mathrm{HCl}$ ( $\mathrm{pH} 7.5$ ), $10 \mathrm{mM} \mathrm{MgCl}_{2}, 10 \mathrm{mM} \mathrm{NH}_{4} \mathrm{Cl}, 20 \mathrm{mM}$ ATP, $1 \mathrm{mM}$, dithiothreitol (DTT), and $3 \mu \mathrm{M}$ AcbM, and incubated at $30^{\circ} \mathrm{C}$ for $12 \mathrm{~h}$. The freeze-dried reaction residue was then dissolved in water with $1 \mathrm{mM}$ DTT and $3 \mu \mathrm{M} \mathrm{AcbO}$ and incubated at $30^{\circ} \mathrm{C}$ for $12 \mathrm{~h}$ to prepare 4 . The reaction was stopped by adding two volumes of methanol, and the supernatant was concentrated by rotary evaporation and freeze-dried. The powder was dissolved in equal volume of water and incubated with $10 \mu \mathrm{M} \mathrm{Zn}^{2+}$, $1 \mathrm{mM} \mathrm{DTT}, 1 \mathrm{mM}$ NADPH, and $3 \mu \mathrm{M}$ AcbL at $30^{\circ} \mathrm{C}$ for $6 \mathrm{~h}$. The reaction mixture with boiled AcbL was set as control. A portion of the reaction mixture was analyzed by HPLC-TOF/MS, and the remaining mixture was further incubated with $3 \mu \mathrm{M}$ AcbJ for $2 \mathrm{~h}$ and detected by GC-QMS.

For the enzymatic assay of AcbN, reaction mixture, containing $25 \mathrm{mM}$ Tris- $\mathrm{HCl}$ (pH 7.5), $10 \mathrm{mM} \mathrm{MgCl}, 1.25 \mathrm{mM} \mathrm{12}, 1 \mathrm{mM} \mathrm{NADPH}$, and $3 \mu \mathrm{M}$ enzyme, was incubated at $30^{\circ} \mathrm{C}$ for $6 \mathrm{~h}$. The reaction mixture with boiled AcbN was set as control. A portion of the reaction mixture was analyzed by HPLC-TOF/MS, and the remaining mixture was further incubated with $3 \mu \mathrm{M}$ AcbJ for $2 \mathrm{~h}$ and detected by GC-QMS. The similar procedure was used to characterize the reverse reaction by replacing 12 and NADPH with 11 and $\mathrm{NADP}^{+}$. To identify the optimal cofactors for the reversible conversion between $\mathbf{1 2}$ and $\mathbf{1 1}$, the oxidation of NADH or NADPH and the reduction of $\mathrm{NAD}^{+}$or $\mathrm{NADP}^{+}$in the corresponding reaction mixtures were monitored by a spectrophotometer (UV-2550, Shimadzu) at $A_{340 \mathrm{~nm}}$ for $6 \mathrm{~min}$.

To examine whether the CFEs of Actinoplanes sp. SE50/110, SE50/110 $\triangle a c b N$ and QQ-3 catalyze the reduction of 12, cells were harvested from 2-day fermentation broth through centrifugation, washed twice, re-suspended in buffer $\mathrm{TN}$, and lysed by sonication. The soluble proteins were separated from the cell debris by centrifugation at $15,777 \times g$ for $10 \mathrm{~min}$ at $4{ }^{\circ} \mathrm{C}$. Subsequently, the reaction mixture containing $25 \mathrm{mM}$ Tris- $\mathrm{HCl}$ (pH 7.5), $10 \mathrm{mM} \mathrm{MgCl}_{2}, 1.25 \mathrm{mM} \mathrm{12}, 1 \mathrm{mM}$ $\mathrm{NADPH}$, and $15 \mathrm{~g} \mathrm{~L}^{-1}$ of crude enzymes was incubated at $30^{\circ} \mathrm{C}$ for $6 \mathrm{~h}$. The reaction mixtures with boiled CFEs were set as controls. A portion of reaction mixture was analyzed by HPLC-TOF/MS, and the remaining mixture was further incubated with $3 \mu \mathrm{M}$ AcbJ for $2 \mathrm{~h}$ and detected by GC-QMS.

In addition, the method for enzymatic assay of the putative NADPH-dependent oxidoreductases was similar with that of AcbN. However, for the oxidoreductases using unknown or unstable cofactors, they were incubated with 12 in the presence of the supernatant of BCF of QQ-3. One enzyme unit (U) is defined as the amount of $\mathbf{1 1}$ in nanomole produced in $1 \mathrm{~min}$.

Purification and identification of the reaction products. For the purification of AcbN-catalyzed product, a $5-\mathrm{mL}$ reaction mixture containing $25 \mathrm{mM}$ Tris- $\mathrm{HCl}$ (pH 7.5), $10 \mathrm{mM} \mathrm{MgCl}_{2}, 1.25 \mathrm{mM} \mathrm{12}, 5 \mathrm{mM} \mathrm{NADPH}$, and $3 \mu \mathrm{M}$ AcbN was incubated at $30^{\circ} \mathrm{C}$ for $6 \mathrm{~h}$. The reaction was stopped by adding two volumes of methanol followed with vigorous vortex to denature the proteins. The mixture was centrifuged at $15,777 \times g$ for $10 \mathrm{~min}$, and the supernatant was concentrated and freeze-dried. The powder was dissolved in 1-mL water and subjected to Sephadex LH-20 column. Fractions containing target compound were pooled. The same method was used to purify the reaction product catalyzed by the CFE of QQ-3 using 12 as substrate. The $1 \mathrm{D}\left({ }^{1} \mathrm{H}\right.$ and $\left.{ }^{13} \mathrm{C}\right)$ and $2 \mathrm{D}\left({ }^{1} \mathrm{H}^{-1} \mathrm{H}\right.$ COSY, HSQC, HMBC, and NOESY) NMR spectra of the purified compounds were collected in $\mathrm{D}_{2} \mathrm{O}$ at $700 \mathrm{MHz}\left({ }^{1} \mathrm{H}\right.$ NMR $)$ and $175 \mathrm{MHz}\left({ }^{13} \mathrm{C}\right.$ NMR) on Bruker AVANCE NEO 700 spectrometer (magnetic field strength with $16.44 \mathrm{~T}$ ).

Feeding experiments. For the feeding experiments, $150 \mu \mathrm{L}$ of seed cultures of Actinoplanes sp. SE50/110 or the derivatives were inoculated to $850-\mu \mathrm{L}$ fermentation medium in 24-well deep well plates by adding $5 \mathrm{mg} \mathrm{L}^{-1}$ of 8 or 9 . After 48 -h incubation on rotary microplate shaker $\left(30^{\circ} \mathrm{C}, 250\right.$ r.p.m.) (Parallel-bioreactor, China), the supernatant of the fermentation broth was diluted for 2 folds and analyzed by HPLC-QQQ/MS.

The workflow for identification of the oxidoreductases. To prepare the cellular proteins of QQ-3, cells were harvested from 2-day fermentation broth by 
centrifugation, washed twice, re-suspended in buffer T ( $25 \mathrm{mM}$ Tris-HCl, $\mathrm{pH} 8.0$, $1 \mathrm{mM}$ DTT), and lysed by sonication. The supernatant was separated from the cell debris by centrifugation at $15,777 \times g$ for $45 \mathrm{~min}$ at $4{ }^{\circ} \mathrm{C}$ and filtered through a $0.5 \mu \mathrm{m}$ membrane.

The fractionation of cellular proteins was performed on AKTA Explorer 100 (GE) with anion exchange chromatography HiTrap Q FF and HiTrap Q HP (GE). The prepacked columns were pre-treated according to the instructions. To enrich and pre-fractionate the target proteins, $70 \mathrm{~mL}$ of crude CFE $\left(22.4 \mathrm{~g} \mathrm{~L}^{-1}\right)$ were loaded to the HiTrap Q FF and eluted at a flow rate of $1 \mathrm{~mL} \mathrm{~min}^{-1}$ by (A) $25 \mathrm{mM}$ Tris- $\mathrm{HCl}(\mathrm{pH} 8.0)$ and (B) $25 \mathrm{mM}$ Tris- $\mathrm{HCl}(\mathrm{pH} 8.0), 1 \mathrm{M} \mathrm{NaCl}$ with the gradient elution procedures: $0-40 \mathrm{~min}, 0 \% \mathrm{~B} ; 40-60 \mathrm{~min}, 10 \% \mathrm{~B} ; 60-80 \mathrm{~min}, 20 \% \mathrm{~B}$; $80-100$ min, $30 \%$ B; $100-120$ min, $40 \%$ B; $120-140$ min, $50 \%$ B; $140-160$ min, $60 \%$ B; $160-180$ min, $70 \%$ B; $180-200$ min 100\% B. These fractions were collected, concentrated, and analyzed for the oxidoreductase activities. Since fractions eluted by $10-40 \%$ B showing enzymatic activity, they were pooled and desalted by $10 \mathrm{kDa}$ concentrator (Millipore Amicon Ultra). For fine fractionation, the pooled fractions were subjected to HiTrap Q HP and separated into 34 fractions with a linear gradient elution by increasing the concentration of B from 10 to $40 \%$ in $120 \mathrm{~min}$. The enzyme activities of these fractions were analyzed in the presence of NADPH or BCF.

The fractions with highest activity and without detectable activity were analyzed by Thermo Scientific ${ }^{m} \mathrm{Q}$ Exactive ${ }^{\mathrm{mx}}$ hybrid quadrupole-Orbitrap mass spectrometer. Among the identified proteins, the annotated oxidoreductases with higher reliability and abundance (Unique PepCount $\geq 4$ ) were chosen and analyzed. The candidates were heterologously expressed in E. coli and purified, and their enzymatic activities were monitored as described above.

\section{Transcriptome sequencing and quantitative real-time PCR analysis. For} transcriptome sequencing of Actinoplanes sp. SE50/110, the cells were harvested from $1-\mathrm{mL}$ fermentation culture at 24,48 , or $72 \mathrm{~h}$ by centrifuging at $4{ }^{\circ} \mathrm{C}$, freezed in liquid nitrogen for $0.5-1 \mathrm{~h}$, and stored at $-80^{\circ} \mathrm{C}$. The RNA extraction, transcriptome sequencing, and data analysis were performed by Shanghai Biotechnology Corporation. The expression of each gene was calculated as fragments per kilobase of exon per megabase of library size.

Because the productivity of $\mathbf{1}$ and transcription of genes in $a c b$ cluster were highest at $48 \mathrm{~h}$ over the fermentation course of Actinoplanes sp. SE50/110 (Supplementary Fig. 55), we took a sample at $48 \mathrm{~h}$ to verify the transcription of related genes. The mycelia were harvested from $1-\mathrm{mL}$ fermentation culture by centrifugation at $15,777 \times g$ for $10 \mathrm{~min}$ at $4{ }^{\circ} \mathrm{C}$, stored in liquid nitrogen for $0.5-1 \mathrm{~h}$, re-suspended with $1 \mathrm{~mL}$ Redzol, and broken by high-speed homogenizer $(65 \mathrm{~Hz}$, $30 \mathrm{~s}$, repeated three times) with glass beads. Then, total RNA of the samples was extracted according to the instruction given in the kit (SBS Genetech). The quality and concentration of total RNA were analyzed by NanoDrop

2000 spectrophotometer. The contaminated DNA was digested by DNase I (Thermo Fisher) according to the instructions. Then, the digested sample was used as a template for PCR amplification using $h r d B-\mathrm{q}-\mathrm{F} / h r d B-\mathrm{q}-\mathrm{R}$ as primers (Supplementary Data 1 ) to verify whether DNA was completely removed. The reverse transcription was performed using RevertAid ${ }^{\mathrm{TM}} \mathrm{H}$ Minus First Strand cDNA Synthesis Kit (Thermo Fisher). The transcription of the related genes was monitored by quantitative real-time PCR using Maxima ${ }^{\mathrm{TM}}$ SYBR Green/ROX qPCR Maxter Mix (Thermo Fisher) ${ }^{58}$. The relative transcription values of the target genes were calculated by the $2^{-\Delta \Delta C t}$ method $^{59}$ with the housekeeping gene $h r d B\left(A C P L \_1268\right)$ as an internal control.

Analysis of enzyme activities in crude CFE. From 2-mL 2-day fermentation broth, mycelia of Actinoplanes sp. SE50/110 or its derivatives were harvested by centrifuged at $4{ }^{\circ} \mathrm{C}$, washed twice, and re-suspended in 0.5 volume pre-cooling buffer ( $25 \mathrm{mM}$ Tris-HCl, pH 8.0, $300 \mathrm{mM} \mathrm{NaCl}, 1 \mathrm{mM}$ DTT). After lysis by sonication, the supernatant was obtained by centrifugation at $15,777 \times g$ for 20 min at $4{ }^{\circ} \mathrm{C}$. Concentrations of the total proteins in the extracts were determined with the Bradford Protein Assay Kit.

For the phosphatase activity analysis, $7 \mu \mathrm{L}$ of crude enzyme solution was added to the mixture $(20 \mu \mathrm{L})$ containing $25 \mathrm{mM}$ Tris- $\mathrm{HCl}(\mathrm{pH} 7.5), 10 \mathrm{mM} \mathrm{MgCl}, 1.25$ $\mathrm{mM} 10$ or 11 , and $1 \mathrm{mM} \mathrm{DTT}$, and then incubated at $30^{\circ} \mathrm{C}$ for $4 \mathrm{~h}$. The reaction was stopped by adding two volumes of methanol and analyzed by GC-QMS.

For the analysis of G-1-PT activity, $2 \mu \mathrm{L}$ of crude enzyme solution was added to the mixture $(20 \mu \mathrm{L})$ containing $50 \mathrm{mM}$ Tris- $\mathrm{HCl}(\mathrm{pH} 7.5), 12 \mathrm{mM} \mathrm{MgCl} 2,24 \mathrm{mM}$ 5, $6 \mathrm{mM}$ dTTP, $0.12 \mathrm{U}$ pyrophosphatase, and $1 \mathrm{mM}$ DTT, and then incubated at $30^{\circ} \mathrm{C}$ for $15 \mathrm{~min}^{60}$. The production of dTDP-D-glucose was analyzed by HPLC$\mathrm{QQQ} / \mathrm{MS}$. One enzyme unit $(\mathrm{U})$ is defined as the amount of dTDP-D-glucose in nanomole produced in $1 \mathrm{~min}$.

For the analysis of 4,6-DH activity, $2 \mu \mathrm{L}$ of crude enzyme solution was added to the mixture $(20 \mu \mathrm{L})$ containing $50 \mathrm{mM}$ Tris- $\mathrm{HCl}(\mathrm{pH} 7.5), 0.4 \mathrm{mM}$ dTDP-D-glucose, $1 \mathrm{mM} \mathrm{NADP}^{+}$, and $1 \mathrm{mM}$ DTT, and then incubated at $30^{\circ} \mathrm{C}$ for $45 \mathrm{~min}^{61}$. The production of dTDP-4-keto-6-deoxy-D-glucose was analyzed by HPLC-QQQ/MS. One enzyme unit $(\mathrm{U})$ is defined as the amount of dTDP-4-keto-6-deoxy-D-glucose in nanomole produced in $1 \mathrm{~min}$. The average specific activity in SE50/110::pSET152 was calculated and set to 1 as standard. The specific activities in related mutants were accordingly calculated.
Analysis of the concentrations of endogenous $\mathbf{1 0}$ and 11. After 2-day fermentation, two samples were, respectively, obtained from $1 \mathrm{~mL}$ of fermentation culture by centrifugation. One sample was washed with $0.25 \mathrm{M}$ hydrochloric acid and dried for biomass measurement, and the other sample was rapidly washed twice with cold $0.9 \% \mathrm{NaCl}$ and put in liquid nitrogen for $0.5-1 \mathrm{~h}$. Then, the sample was re-suspended in equal volume of water, lysed by sonication, and rapidly mixed with 1 -mL cold methanol. The supernatant was separated from the cell debris by centrifugation at $15,777 \times g$ for $20 \mathrm{~min}$ at $4{ }^{\circ} \mathrm{C}$ and freeze-dried. The powder was resuspended by $100 \mu \mathrm{L}$ methanol/water solution (50:50, v/v), centrifuged at $15,777 \times$ $g$ for $20 \mathrm{~min}$ and subjected to HPLC-QQQ/MS analysis. The concentrations of $\mathbf{1 0}$ and $\mathbf{1 1}$ were calculated according to the biomass. The concentrations of $\mathbf{1 0}$ or 11 in SE50/110::pSET152 or Actinoplanes sp. SE50/110 were set to 1 as standards, and those of related mutants were accordingly calculated.

Reporting summary. Further information on research design is available in the Nature Research Reporting Summary linked to this article.

\section{Data availability}

Data supporting the findings of this work are available within the paper and its Supplementary Information files. A reporting summary for this article is available as a Supplementary Information file. The datasets generated and analyzed during the current study are available from the corresponding author upon request. The source data underlying Figs. 2c, 3-5, 6b, c, e, f, and 7 are provided as a Source Data file. The sequences for all genes described in this manuscript are available in the GenBank databases under the accession numbers Y18523.4, CP003170.1, СР003275.1, CP000480.1, CP002496.1, and AP009048.1.

Received: 1 May 2019; Accepted: 23 February 2020; Published online: 19 March 2020

\section{References}

1. Wehmeier, U. F. \& Piepersberg, W. Biotechnology and molecular biology of the a-glucosidase inhibitor acarbose. Appl. Microbiol. Biotechnol. 63, 613-625 (2004)

2. Asamizu, S. Biosynthesis of nitrogen-containing natural products, $\mathrm{C}_{7} \mathrm{~N}$ aminocyclitols and bis-indoles, from actinomycetes. J. Agric. Chem. Soc. Jpn. 81, 871-881 (2017)

3. Ahr, H. J. et al. Pharmacokinetics of acarbose. Part I: absorption, concentration in plasma, metabolism and excretion after single administration of $\left[{ }^{14} \mathrm{C}\right]$ acarbose to rats, dogs and man. Arzneimittelforschung 39, 1254-1260 (1989).

4. Juergen, B., Michael, S. \& Ulrich, S. Osmotically controlled fermentation process for the preparation of acarbose. US patent 6,130,072 (2000).

5. Feng, Z. H., Wang, Y. S. \& Zheng, Y. G. A new microtiter plate-based screening method for microorganisms producing $a$-amylase inhibitors. Biotechnol. Bioproc. E 16, 894-900 (2011).

6. Cho, N. H. et al. IDF Diabetes Atlas: global estimates of diabetes prevalence for 2017 and projections for 2045. Diabetes Res. Clin. Pract. 138, 271 (2018).

7. Schwientek, P. et al. The complete genome sequence of the acarbose producer Actinoplanes sp. SE50/110. BMC Genomics 13, 112 (2012).

8. Xie, H., Zhao, Q., Zhang, X., Kang, Q. \& Bai, L. Comparative functional genomics of the acarbose producers reveals potential targets for metabolic engineering. Synth. Syst. Biotechnol. 4, 49-56 (2019).

9. Schwientek, P. et al. Comparative RNA-sequencing of the acarbose producer Actinoplanes sp. SE50/110 cultivated in different growth media. J. Biotechnol. 167, 166-177 (2013).

10. Wendler, S. et al. Comparative proteome analysis of Actinoplanes sp. SE50/110 grown with maltose or glucose shows minor differences for acarbose biosynthesis proteins but major differences for saccharide transporters. J. Proteom. 131, 140-148 (2016).

11. Gren, T. et al. Genetic engineering in Actinoplanes sp. SE50/110development of an intergeneric conjugation system for the introduction of actinophage-based integrative vectors. J. Biotechnol. 232, 79-88 (2016).

12. Wolf, T. et al. Targeted genome editing in the rare actinomycete Actinoplanes sp. SE50/110 by using the CRIPSR/Cas9 system. J. Biotechnol. 231, 122-128 (2016).

13. Zhao, Q., Xie, H., Peng, Y., Wang, X. \& Bai, L. Improving acarbose production and eliminating the by-product component $C$ with an efficient genetic manipulation system of Actinoplanes sp. SE50/110. Synth. Syst. Biotechnol. 2, 302-309 (2017).

14. Lee, J. W. et al. Systems metabolic engineering of microorganisms for natural and non-natural chemicals. Nat. Chem. Biol. 8, 536-546 (2012).

15. Pickens, L. B., Tang, Y. \& Chooi, Y. H. Metabolic engineering for the production of natural products. Annu. Rev. Chem. Biomol. 2, 211 (2011). 
16. Bilyk, O. \& Luzhetskyy, A. Metabolic engineering of natural product biosynthesis in actinobacteria. Curr. Opin. Biotechnol. 42, 98-107 (2016).

17. Crueger, A., Piepersberg, W., Distler, J. \& Stratmann, A. Acarbose biosynthesis genes from Actinoplanes sp., process for the isolation thereof and the use thereof. US patent 5,753,501 (1998).

18. Licht, A. et al. Crystal structures of the bacterial solute receptor AcbH displaying an exclusive substrate preference for $\beta$-D-galactopyranose. J. Mol. Biol. 406, 92-105 (2011).

19. Mahmud, T. et al. Biosynthetic studies on the $\alpha$-glucosidase inhibitor acarbose in Actinoplanes sp.: 2-epi-5-epi-valiolone is the direct precursor of the valienamine moiety. J. Am. Chem. Soc. 121, 6973-6983 (1999).

20. Stratmann, A. et al. The AcbC protein from Actinoplanes species is a $\mathrm{C}_{7^{-}}$ cyclitol synthase related to 3-dehydroquinate synthases and is involved in the biosynthesis of the a-glucosidase inhibitor acarbose. J. Biol. Chem. 274, 10889-10896 (1999).

21. Zhang, C. S. et al. Biosynthesis of the $\mathrm{C}_{7}$-cyclitol moiety of acarbose in Actinoplanes species SE50/110. 7-O-phosphorylation of the initial cyclitol precursor leads to proposal of a new biosynthetic pathway. J. Biol. Chem. 277, 22853-22862 (2002).

22. Zhang, D., Zhao, Q., Jiang, M., Kang, Q. \& Bai, L. Biosynthetic pathway of deoxyaminosugar moiety in acarbose from Actinoplanes sp. SE50/110. Acta Microbiol. Sin. 60, 118-134 (2019).

23. Lee, S., Sauerbrei, B., Niggemann, J. \& Egelkrout, E. Biosynthetic studies on the a-glucosidase inhibitor acarbose in Actinoplanes sp.: source of the maltose unit. J. Antibiot. 50, 954-960 (1997).

24. Alanzi, A. R., Demessie, A. A. \& Mahmud, T. Biosynthesis and metabolic engineering of pseudo-oligosaccharides. Emerg. Top. Life Sci. 2, 405-417 (2018).

25. Minagawa, K. et al. ValC, a New type of $\mathrm{C}_{7}$-cyclitol kinase involved in the biosynthesis of the antifungal agent validamycin A. ChemBioChem 8, 632-641 (2010).

26. Kumar, M. et al. Functional genome screening to elucidate the colistin resistance mechanism. Sci. Rep. 6, 23156 (2016).

27. $\mathrm{Xu}, \mathrm{H}$. et al. Alternative epimerization in $\mathrm{C}_{7} \mathrm{~N}$-aminocyclitol biosynthesis is catalyzed by ValD, a large protein of the vicinal oxygen chelate superfamily. Chem. Biol. 16, 567-576 (2009).

28. Shi, X., Sha, S., Liu, L., Xin, L. \& Ma, Y. A 96-well microtiter plate assay for high-throughput screening of Mycobacterium tuberculosis dTDP-D-glucose 4,6-dehydratase inhibitors. Anal. Biochem. 498, 53-58 (2016)

29. Aguirreramírez, M., Medina, G., Gonzálezvaldez, A., Grossobecerra, V. \& Soberónchávez, G. The Pseudomonas aeruginosa rmlBDAC operon, encoding dTDP-L-rhamnose biosynthetic enzymes, is regulated by the quorum-sensing transcriptional regulator RhlR and the alternative sigma factor $\sigma^{\mathrm{S}}$. Microbiology 158, 908-916 (2012).

30. Gerratana, B., Cleland, W. W. \& Frey, P. A. Mechanistic roles of Thr134, Tyr160, and Lys164 in the reaction catalyzed by dTDP-glucose 4,6dehydratase. Biochemistry (Mosc.) 40, 9187 (2001).

31. Sha, S., Zhou, Y., Xin, Y. \& Ma, Y. Development of a colorimetric assay and kinetic analysis for Mycobacterium tuberculosis D-glucose-1-phosphate thymidylyltransferase. J. Biomol. Screen. 17, 252-257 (2012).

32. Alphey, M. S. et al. Allosteric competitive inhibitors of the glucose-1phosphate thymidylyltransferase (RmlA) from Pseudomonas aeruginosa. ACS Chem. Biol. 8, 387-396 (2013).

33. Zuccotti, S. et al. Kinetic and crystallographic analyses support a sequentialordered bi bi catalytic mechanism for Escherichia coli glucose-1-phosphate thymidylyltransferase. J. Mol. Biol. 313, 831-843 (2001).

34. Flatt, P. M., Wu, X., Perry, S. \& Mahmud, T. Genetic insights into pyralomicin biosynthesis in Nonomuraea spiralis IMC A-0156. J. Nat. Prod. 76, 939-946 (2013).

35. Choi, W. S. et al. Genetic organization of the putative salbostatin biosynthetic gene cluster including the 2-epi-5-epi-valiolone synthase gene in Streptomyces albus ATCC 21838. Appl. Microbiol. Biotechnol. 80, 637-645 (2008).

36. Rockser, Y. \& Wehmeier, U. F. The gac-gene cluster for the production of acarbose from Streptomyces glaucescens GLA.O-identification, isolation and characterization. J. Biotechnol. 140, 114-123 (2009).

37. Zhang, C. S. et al. Identification of a 1-epi-valienol 7-kinase activity in the producer of acarbose, Actinoplanes sp. SE50/110. FEBS Lett. 540, 53-57 (2003).

38. Ekaterina, K. et al. Functional diversity of haloacid dehalogenase superfamily phosphatases from Saccharomyces cerevisiae: biochemical, structural, and evolutionary insights. J. Biol. Chem. 290, 18678-18698 (2015).

39. Kadner, R. J., Murphy, G. P. \& Stephens, C. M. Two mechanisms for growth inhibition by elevated transport of sugar phosphates in Escherichia coli. J. Gen. Microbiol. 138, 2007-2014 (1992).

40. Schaffert, L. et al. Evaluation of vector systems and promoters for overexpression of the acarbose biosynthesis gene $a c b C$ in Actinoplanes sp. SE50/110. Microb. Cell Fact. 18, 114 (2019).
41. $\mathrm{Wu}, \mathrm{Z}$. et al. Improving gentamicin $\mathrm{B}$ and gentamicin $\mathrm{Cla}$ production by engineering the glycosyltransferases that transfer primary metabolites into secondary metabolites biosynthesis. Microbiol. Res. 203, 40-46 (2017).

42. Ekaterina, K. et al. Genome-wide analysis of substrate specificities of the Escherichia coli haloacid dehalogenase-like phosphatase family. J. Biol. Chem. 281, 36149-36161 (2006).

43. Agne, T., Mark George, T. \& Joachim, J. Escherichia coli kduD encodes an oxidoreductase that converts both sugar and steroid substrates. Appl. Microbiol. Biotechnol. 98, 5471-5485 (2014).

44. Yun, E. J. et al. Promiscuous activities of heterologous enzymes lead to unintended metabolic rerouting in Saccharomyces cerevisiae engineered to assimilate various sugars from renewable biomass. Biotechnol. Biofuels 11, 140 (2018).

45. Copley, S. D. Shining a light on enzyme promiscuity. Curr. Opin. Struct. Biol. 47, 167-175 (2017).

46. Chae, T. U., Choi, S. Y., Kim, J. W., Ko, Y. S. \& Lee, S. Y. Recent advances in systems metabolic engineering tools and strategies. Curr. Opin. Biotechnol. 47, 67-82 (2017)

47. Zhao, X., Park, S. Y., Yang, D. \& Lee, S. Y. Synthetic biology for natural compounds. Biochemistry (Mosc.) 58, 1454-1456 (2019).

48. Ren, F., Chen, L., Xiong, S. \& Tong, Q. Enhanced acarbose production by Streptomyces M37 using a two-stage fermentation strategy. PLoS ONE 12, e0166985 (2017).

49. Li, K. T., Zhou, J., Wei, S. J. \& Cheng, X. An optimized industrial fermentation processes for acarbose production by Actinoplanes sp. A56. Bioresour. Technol. 118, 580-583 (2012).

50. Sun, L. H., Li, M. G., Wang, Y. S. \& Zheng, Y. G. Significantly enhanced production of acarbose in fed-batch fermentation with the addition of $S$ adenosylmethionine. J. Microbiol. Biotechnol. 22, 826-831 (2012).

51. Wu, X., Flatt, P., Xu, H. \& Mahmud, T. Biosynthetic gene cluster of cetoniacytone $\mathrm{A}$, an unusual aminocyclitol from the endosymbiotic bacterium Actinomyces sp. Lu 9419. ChemBioChem 10, 304-314 (2010).

52. Li, X., Wang, W. \& Yang, K. An engineered strong promoter for Streptomycetes. Appl. Environ. Microbiol. 79, 4484-4492 (2013).

53. Qi, Z., Kang, Q., Jiang, C., Han, M. \& Bai, L. Engineered biosynthesis of pimaricin derivatives with improved antifungal activity and reduced cytotoxicity. Appl. Microbiol. Biotechnol. 99, 6745-6752 (2015).

54. Bibb, M. J., Janssen, G. R. \& Ward, J. M. Cloning and analysis of the promoter region of the erythromycin resistance gene (ermE) of Streptomyces erythraeus. Gene 41, E357-E368 (1985).

55. Bierman, M. et al. Plasmid cloning vectors for the conjugal transfer of DNA from Escherichia coli to Streptomyces spp. Gene 116, 43-49 (1992).

56. Yang, Z. et al. Identification and functional analysis of the gene ste9 involving in ebosin biosynthesis from Streptomyces sp. 139. FEMS Microbiol. Lett. 350, 257-264 (2014).

57. Verma, J. P. Data Analysis in Management with SPSS Software (Springer, 2013).

58. Wang, X., Ning, X., Zhao, Q., Kang, Q. \& Bai, L. Improved PKS gene expression with strong endogenous promoter resulted in geldanamycin yield increase. Biotechnol. J. 12, 1700321 (2017).

59. Livak, K. J. \& Schmittgen, T. D. Analysis of relative gene expression data using real-time quantitative PCR and the $2^{-\Delta \Delta C T}$ method. Methods 25, 402-408 (2001).

60. Lee, H. C. et al. Cloning and expression of the glucose-1-phosphate thymidylyltransferase gene (gerD) from Streptomyces sp. GERI-155. Mol. Cells 17, 274-280 (2004).

61. Lee, H. et al. Cloning, expression, and biochemical characterization of dTDPglucose 4,6-dehydratase gene (gerE) from Streptomyces sp. GERI-155. J. Microbiol. Biotechnol. 14, 576-583 (2004).

\section{Acknowledgements}

We thank Dr. Weishan Wang and the late Prof. Keqian Yang for providing kasOp* promoter and Xingzhi Jiao, Fucai Ren, and Prof. Yuemao Shen for help with NMR data processing. We also thank the Core Facility and Technical Service Center for SLSB in SJTU for providing services, and thank Prof. Taifo Mahmud and Prof. Yi Tang for revising the manuscript. This work is supported by National Natural Science Foundation of China (Grants 31830104, 21661140002, U1703236), National Key R\&D Program of China (2019YFA0905400), and Science and Technology Commission of Shanghai Municipality (Grant 17JC1403600).

\section{Author contributions}

Q.Z., L.Z., and L.B. designed the research; Q.Z., Y.L., X.Z., and D.Z. performed the research; Q.Z., Y.L., and Q.K. analyzed data; and Q.Z., L.Z., L.B., and Z.D. wrote the manuscript.

\section{Competing interests}

The authors declare no competing interests. 


\section{Additional information}

Supplementary information is available for this paper at https://doi.org/10.1038/s41467020-15234-8

Correspondence and requests for materials should be addressed to L.B.

Peer review information Nature Communications thanks Jörn Kalinowski, Jürgen Rohr and Udo Wehmeier for their contribution to the peer review of this work.

Reprints and permission information is available at http://www.nature.com/reprints

Publisher's note Springer Nature remains neutral with regard to jurisdictional claims in published maps and institutional affiliations. (c) (i) Open Access This article is licensed under a Creative Commons Attribution 4.0 International License, which permits use, sharing, adaptation, distribution and reproduction in any medium or format, as long as you give appropriate credit to the original author(s) and the source, provide a link to the Creative Commons license, and indicate if changes were made. The images or other third party material in this article are included in the article's Creative Commons license, unless indicated otherwise in a credit line to the material. If material is not included in the article's Creative Commons license and your intended use is not permitted by statutory regulation or exceeds the permitted use, you will need to obtain permission directly from the copyright holder. To view a copy of this license, visit http://creativecommons.org/ licenses/by/4.0/.

(C) The Author(s) 2020 